\title{
A Direct Construction of Optimal ZCCS With Maximum Column Sequence PMEPR Two for MC-CDMA System
}

\author{
Palash Sarkar, and Sudhan Majhi
}

\begin{abstract}
Multicarrier code-division multiple-access (MCCDMA) combines an orthogonal frequency division multiplexing (OFDM) modulation and a code-division multiple-access (CDMA) scheme to exploits the benefits of both the technologies. The high peak-to-mean envelope power ratio (PMEPR) is a considerable problem in MC-CDMA system. However, the problem can be addressed by utilizing complete complementary codes (CCCs) in MC-CDMA system. But the set size upper bound of CCC does not allow the system to support large number of users for a given number of subcarriers in the system. In a CCC and Zcomplementary code set (ZCCS) based asynchronous MC-CDMA system, the PMEPR is determined by column sequence PMEPR of the codes. In order to suuport a large number of users with low column sequence PMEPR, in this paper, we have proposed a new optimal ZCCS with larger set size. The code is constructed using Boolean function approach, i.e., by a direct construction method. The number of constituent sequences in ZCCS is the same as the number of subcarriers in MC-CDMA. So, large size ZCCS for large number of users in MC-CDMA can be constructed through a rapid hardware generation. The proposed ZCCS has mximum column sequence PMEPR of 2 and it achieves the theoretical upper bound of optimality. Our proposed construction can also generate inter-group complementary (IGC) code set for MCCDMA with the same PMEPR. This work also establishes a link from ZCCS and IGC code set to higher-order $(\geq 2)$ Reed-Muller (RM) code.
\end{abstract}

Index Terms-Complementary code (CC), complete complementary code (CCC), multicarrier code-division multiple-access (MC-CDMA), generalized Boolean function (GBF), inter-group complementary (IGC) code set, Reed-Muller (RM) codes, Zcomplementary code set (ZCCS), zero correlation zone (ZCZ)

\section{INTRODUCTION}

Multicarrier code-division multiple-access (MC-CDMA) is a most promising technology for fifth generation (5G) and beyond wireless communication. It has brilliant features as it combines multicarrier modulation and multiplexing technique. However, it suffers from high peak-to-mean envelope power ratio (PMEPR) problem. The high PMEPR value problem in MC-CDMA system can be compensated by employing proper spreading codes which provide low column sequence PMEPR. Because of suitable auto- and cross-correlation properties, such spreading codes [1]-[8] are also used to deal multiple access interference (MAI) and multipath interference (MPI) besides PMEPR problem.

Palash Sarkar is with Department of Mathematics and Sudhan Majhi is with the Department of Electrical Engineering, Indian Institute of Technology Patna, India, e-mail: palash.pma15@iitp.ac.in; smajhi@iitp.ac.in.
In this context, we introduce Golay complementary pair (GCP), complete complementary code (CCC), and ZCCS. A pair of sequences, with the sum of their aperiodic autocorrelation function (AACF) to zero for all nonzero time shift, called GCP [9]. Sequences of a GCP is known as Golay sequences. The concept of complementary code (CC) was introduced by Tseng and Liu in [10] by extending the idea of GCP. The sum of AACFs of the sequences in a CC become zero for all out of phase shift. In 1999, Davis et al. proposed a constrcution of GCP in [11], known as Golay-Davis-Jedweb (GDJ), by using second-order generalized Boolean function (GBF) and provided a link between their GCPs and ReedMuller (RM) code. Later, Paterson et al. proffered a construction of $\mathrm{CC}$ by using graph and second-order RM code in [12] and the work is generalized by Schmidth by using higher-order RM in [13]. Paterson's idea of CCs were extended to CCC by Rathinakumar et al. in [1] by using second-order GBF. A set of CCs with ideal cross-correlation properties is said to be $\mathrm{CCC}$ if the number of $\mathrm{CCs}$ is equal to the number sequences in each CC. A construction of CCC were introduced in [14] where it was shown that the column sequence PMEPR of a CCC based MC-CDMA system can have at most 2 unlike the $\mathrm{CCC}$ introduced in [1].

ZCCS has the same correlation properties as CCC inside a zone, called zero correlation zone (ZCZ). As compared with CCC, ZCCS has much larger set size [15] which allows a ZCCS based MC-CDMA system to support a large number of users unlike CCC based MC-CDMA system where number of subcarriers is equal to the number of users. Having the $\mathrm{ZCZ}$ properties, ZCCS is used to mitigate MAI for received multiuser quasi-synchronous signals within the ZCZ width [16]. In 2007, Fan et al. [17] introduced binary ZCCS and it is generalized to pairwise ZCCS by Feng et al. [18] in 2008. In 2019, a construction of ZCCS has been introduced by Palash et al. in [3] by associating it with second-order RM code and graph. Another construction of ZCCS has been reported in [4] by using second-order GBFs. In 2015, a construction of ZCCS which has maximum column sequence PMEPR of 2, was introduced by Li et al. in [5]. The construction is based on Golay sequences and orthogonal matrix. But this construction is not a direct construction and it may not be advantageous for the hardware generation of long ZCCSs. To reduce high PMEPR problem and in order to support a large number of users in a MC-CDMA system, the aim of this paper is to provide a direct construction of a new ZCCS based on which a MC-CDMA system can have PMEPR of at most 2. 
A ZCCS is known as inter-group complementary (IGC) code set when it is divided into numerous distinct code groups with the properties that the AACF of each code is ideal within the $\mathrm{ZCZ}$ width. The aperiodic cross-correlation function (ACCF) of two disjoint codes drawn from the same code group is also ideal inside the $\mathrm{ZCZ}$ width. The ACCF of two codes drawn from two different code groups is zero for all time shifts. In 2008, Li et al. proposed a construction of IGC code set based on CCCs in [19]. Their code assignment algorithm shows that the CDMA systems employing the IGC codes (IGC-CDMA) outperform traditional CDMA with respect to bit error rate (BER). The ZCZ width of IGC code set in [19] depends on the length of constituent sequences of CCs and the construction is not direct. Recently, a direct construction of IGC code set has introduced in [20] by using second-order GBFs. However, the constructions given in [19], [20] cannot provide a tight column sequence PMEPR as the column sequnce PMEPRs of IGC code sets from both of the constructions is upper bounded by the number of constituent sequences which motivate us to provide a direct constrcution of IGC code set with maximum column sequence PMEPR 2.

In this paper, we first propose a direct construction of ZCCS by using higher-order $(\geq 2)$ GBFs. The maximum column sequence PMEPR of our proposed ZCCS based MC-CDMA system is 2 unlike the ZCCS given in [3], [4]. Then we show that our propose ZCCS can also generate IGC code set with maximum column sequence PMEPR of IGC based MCCDMA is 2 which make our construction more efficient than existing IGC code set construction. Our propose construction establish a relation of ZCCS and IGC code set with higher order $(\geq 2)$ RM code. We also relate our constructions with graph. Specially, we have shown that our propose construction generates ZCCS corresponding to a GBF if the graphs of all possible restrictions of the GBF over some fixed specific variables, contain a path and some fixed isolated vertices. The construction generates IGC code set if the GBF does not contain a term which is associated with the restricted variables and the variables which appear as isolated vertices in the grpahs of restricted Boolean functions.

The paper is arranged as follows. In Section II, some definitions and useful notations are presented. A construction of ZCCS with maximum column sequence PMEPR 2 has been presented in Section III. In Section IV, a construction IGC code set with maximum column sequence PMEPR 2 is presented. We compare our proposed construction with existing construction in Section V. Finally, we conclude our proposed constrcution in Section VI.

\section{PRELIMINARY}

\section{A. Definitions of Correlations and Sequences}

Let $\mathbf{a}=\left(a_{0}, a_{1}, \ldots, a_{L-1}\right)$ and $\mathbf{b}=\left(b_{0}, b_{1}, \ldots, b_{L-1}\right)$ be two complex-valued sequences of equal length $L$. For an integer $\tau$, define

$$
\mathscr{C}(\mathbf{a}, \mathbf{b})(\tau)= \begin{cases}\sum_{i=0}^{L-1-\tau} a_{i+\tau} b_{i}^{*}, & 0 \leq \tau<L, \\ \sum_{l=0}^{L+\tau-1} a_{i} b_{i-\tau}^{*}, & -L<\tau<0, \\ 0, & \text { otherwise }\end{cases}
$$

and $\mathscr{A}(\mathbf{b})(\tau)=\mathscr{C}(\mathbf{b}, \mathbf{b})(\tau)$. The following functions $\mathscr{C}(\mathbf{a}, \mathbf{b})$ and $\mathscr{A}(\mathbf{b})$ are called ACCF of $\mathbf{a}$ and $\mathbf{b}$, and AACF of $\mathbf{b}$ respectively. Let $\mathbf{C}=\left\{C_{0}, C_{1}, \ldots, C_{K-1}\right\}$ where

$$
C_{\mu}=\left[\begin{array}{c}
\mathbf{a}_{0}^{\mu} \\
\mathbf{a}_{1}^{\mu} \\
\vdots \\
\mathbf{a}_{M-1}^{\mu}
\end{array}\right]_{M \times L}=\left[\begin{array}{llll}
\mathbf{d}_{0}^{\mu} & \mathbf{d}_{1}^{\mu} & \cdots & \mathbf{d}_{L-1}^{\mu}
\end{array}\right],
$$

where $\mathbf{a}_{p}^{\mu}(0 \leq p \leq M-1,0 \leq \mu \leq K-1)$ is the $p$ th row sequence or $p$ th constituent sequence of $C_{\mu}$ and $\mathbf{d}_{e}^{\mu}(0 \leq e \leq$ $L-1)$ is the eth column sequence of $C_{\mu}$. For $C_{\mu}, C_{\nu} \in \mathbf{C}$ $(0 \leq \mu, \nu \leq K-1)$, the ACCF of $C_{\mu}$ and $C_{\nu}$ is defined by

$$
\mathscr{C}\left(C_{\mu}, C_{\nu}\right)(\tau)=\sum_{p=0}^{M-1} \mathscr{C}\left(\mathbf{a}_{p}^{\mu}, \mathbf{a}_{p}^{\nu}\right)(\tau) .
$$

Definition 1: $\mathbf{C}$ is called CCC if $K=M$ and it satisfies the following properties:

$$
\mathscr{C}\left(C_{\mu}, C_{\nu}\right)(\tau)= \begin{cases}L M, & \tau=0, \mu=\nu \\ 0, & 0<|\tau|<L, \mu=\nu ; \\ 0, & |\tau|<L, \mu \neq \nu .\end{cases}
$$

The code $C_{\mu}(0 \leq \mu \leq K-1)$, is called CC and it is called GCP if it contains a pair of sequences.

Definition 2: $\mathbf{C}$ is said to be ZCCS and we denote it by $(K, Z)-\mathrm{ZCCS}_{M}^{L}$ if it satisfies the following properties:

$$
\mathscr{C}\left(C_{\mu}, C_{\nu}\right)(\tau)= \begin{cases}L M, & \tau=0, \mu=\nu, \\ 0, & 0<|\tau|<Z, \mu=\nu, \\ 0, & |\tau|<Z, \mu \neq \nu,\end{cases}
$$

where $Z$ is called $\mathrm{ZCZ}$ width.

Definition 3: Let $\mathbf{C}$ can be expressed as the union of $M$ distinct code groups $\mathcal{I}_{g}(g=0,1, \ldots, M-1)$ where each code group is a collection of $K / M$ codes and $K=M L / Z$. C is said to be IGC code set and denoted by $\mathcal{I}(K, M, L, Z)$ if it satisfies the following properties:

$$
\mathscr{C}\left(C_{\mu}, C_{\nu}\right)(\tau)= \begin{cases}M L, & \tau=0, \mu=\nu, \\ 0, & 0<|\tau|<Z, \mu=\nu, \\ 0, & |\tau|<Z, \mu \neq \nu, C_{\mu}, C_{\nu} \in \mathcal{I}_{g}, \\ 0, & |\tau|<L, C_{\mu} \in \mathcal{I}_{g_{1}}, C_{\nu} \in \mathcal{I}_{g_{2}}, g_{1} \neq g_{2}, \\ \text { others, } & \text { otherwise. }\end{cases}
$$

\section{B. Peak-to-Mean Envelope Power Ratio (PMEPR)}

Let $\mathbf{A}=\left(A_{0}, A_{1}, \ldots, A_{M-1}\right)$ be a complex valued sequence of length $M$. For a multi-carrier system with $M$ subcarriers, the time domain multi-carrier signal can be written as [14]

$$
S(\mathbf{A})(t)=\sum_{j=0}^{L-1} A_{j} e^{2 \pi \sqrt{-1} j t},
$$

where the carrier spacing has been normalized to 1 and $\mathbf{A}$ is spreaded over $M$ subcarriers. Denote $P(\mathbf{a})(t)=|S(\mathbf{a})(t)|^{2}$. 
The PMEPR of a polyphase sequence $\mathbf{A}$ under the multi-carrier modulation is defined as

$$
\operatorname{PMEPR}(\mathbf{a})=\frac{1}{M} \sup _{0 \leq t<1} P(\mathbf{A})(t) .
$$

Let $C_{\mu}$ be a code from the ZCCS $\mathbf{C}$ which is defined in Definition 3. In a ZCCS based MC-CDMA system, $\mathbf{a}_{\nu}^{\mu}$ is spread in $\mu$ th subcarrier over $L$ chip-slots and $\mathbf{d}_{\nu^{\prime}}^{\mu}$ is spread in the $\nu^{\prime}$ th chip-slot over $M$ subcarriers. The PMEPR of $C_{\mu}$ is given by

$$
\operatorname{PMEPR}\left(C_{\mu}\right)=\sup _{0 \leq \nu^{\prime}<L} \operatorname{PMEPR}\left(\mathbf{d}_{\nu^{\prime}}^{\mu}\right) .
$$

A CCC based MC-CDMA system transmitter structure is given by Liu et al. in [14]. A ZCCS based MC-CDMA is given in [5] and QCSS based MC-CDMA is given in [21].

\section{Generalized Boolean Functions and Graphs}

There are $2^{m}$ distinct monomials which are of degree $0,1, \ldots, m$ over the variables $x_{0}, x_{1}, \ldots, x_{m-1}$. If $\mathcal{S}_{r}$ is the set of all monomials of degree at most $r, \mathcal{S}_{r}$ can be expressed as

$$
\begin{aligned}
\mathcal{S}_{r}=\left\{x_{\alpha_{1}} x_{\alpha_{2}} \cdots x_{\alpha_{k}}: 0 \leq k \leq r\right. \\
\left.0 \leq \alpha_{1}<\alpha_{2}<\cdots<\alpha_{k} \leq m-1\right\}
\end{aligned}
$$

where $\mathcal{S}_{r}$ contains $\sum_{\alpha=0}^{r}\left(\begin{array}{c}m \\ \alpha\end{array}\right)$ distinct monomials of degree 0 to $r(0 \leq r \leq m)$. A $r$ th degree GBF $f$ of $m$ variables $x_{0}, x_{1}, \ldots, x_{m-1}$ over $\mathbb{Z}_{q}$ can uniquely be expressed as a linear combination of monomials from the set $\mathcal{S}_{r}$ with $\mathbb{Z}_{q^{-}}$ valued coefficients provided that the coefficient of at least one of the $r$ th order monomials is nonzero. For a second-order GBF $f$, the graph of $f$ is denoted by $G(f)$ which contains a edge between the vertices $x_{i}$ and $x_{j}$ if there is a term $q_{i, j} x_{i} x_{j}$ $\left(0 \leq i<j \leq m-1, q_{i, j} \neq 0\right)$ in the expression of $f$. The complex-valued sequence corresponding to $f$ is expressed as follows:

$$
\psi(f)=\left(\omega^{f_{0}}, \omega^{f_{1}}, \ldots, \omega^{f_{2} m_{-1}}\right),
$$

where $f_{i}=f\left(i_{0}, i_{1}, \ldots, i_{m-1}\right), \omega=\exp (2 \pi \sqrt{-1} / q), q(\geq 2)$ is an even number, and $\left(i_{0}, i_{1}, \ldots, i_{m-1}\right)$ is the binary vector representation of $i$. Below some notations are presented for better presentation of the paper:

- $\tilde{f}$ denotes $f\left(1-x_{0}, 1-x_{1}, \ldots, 1-x_{m-1}\right)$.

- $\bar{x}$ denotes the binary complement of $x \in\{0,1\}$.

- $\mathbf{a}^{*}$ is the complex conjugate of a complex-valued vector a.

- $J=\left\{j_{0}, j_{1}, \ldots, j_{k-1}\right\}(\subset\{0,1, \ldots, m-1\})$.

- $\mathbf{x}_{J}=\left(x_{j_{0}}, x_{j_{1}}, \ldots, x_{j_{k-1}}\right)$.

- $\mathbf{c}=\left(c_{0}, c_{1}, \ldots, c_{k-1}\right) \in\{0,1\}^{k}$.

Consider the function $\left.f\right|_{x_{j}=c}$, obtained by substituting $x_{j}=$ $c$ in $f$, be equivalent to the graph obtained by deleting the vertex $x_{j}$ and all the edges associated with $x_{j}$ from $G(f)$. Similarly, $G\left(\left.f\right|_{\mathbf{x}_{J}=\mathbf{c}}\right)$ is obtained by deleting the vertices $x_{j_{0}}, x_{j_{1}}, \ldots, x_{j_{k-1}}$ from $G(f)$. The $i$ th component of the complex-valued sequence $\psi\left(\left.f\right|_{\mathbf{x}=\mathbf{c}}\right)$ is denoted by $\omega^{f\left(i_{0}, i_{1}, \ldots, i_{m-1}\right)}$ if $i_{j_{\alpha}}=c_{\alpha}$ for each $0 \leq \alpha<k$ and equal to zero otherwise.
A second-order GBF $f$ can be expressed as

$$
f=Q+\sum_{i=0}^{m-1} g_{i} x_{i}+g^{\prime}
$$

where $Q$ is the quadratic form present in $f$ and $g^{\prime}, g_{i} \in \mathbb{Z}_{q}$. For more details, readers can go through [1], [3].

Definition 4 (Reed-Muller Code): A set of sequences which are obtained from the GBFs of $m$ variables $x_{0}, x_{1}, \ldots, x_{m-1}$ of order no greater than $r$ over $\mathbb{Z}_{q}$ is said to be $r$ th order RM code over $\mathbb{Z}_{q}$ and is denoted by $\operatorname{RM}_{q}(r, m) . \operatorname{RM}_{q}(r, m)$ is said to be the $r$ th order RM code

\section{Existing Constrcutions of CC, CCC, and ZCCS}

Some lemmas has been presented in this subsection and we also introduce some notations which will be used for our proposed constructions.

Lemma 1 ( [12]): Let $f, g$ are two GBFs. Assume $\mathbf{d}=$ $\left(d_{0}, d_{1}, \ldots, d_{k-1}\right) \in\{0,1\}^{k}$ and $\left\{i_{0}, i_{1}, \cdots, i_{l-1}\right\}$ be a set of $l$ indices such that $0 \leq i_{0}<i_{1}<\cdots<i_{l-1}<m$ and has no intersection with $J$. Let $\mathbf{y}=\left(x_{i_{0}}, x_{i_{1}}, \ldots, x_{i_{l-1}}\right)$, then

$$
\begin{aligned}
\mathscr{C} & \left(\psi\left(\left.f\right|_{\mathbf{x}_{J}=\mathbf{c}}\right), \psi\left(\left.g\right|_{\mathbf{x}_{J}=\mathbf{d}}\right)\right)(\tau) \\
& =\sum_{\mathbf{c}_{1}, \mathbf{c}_{2}} \mathscr{C}\left(\psi\left(\left.f\right|_{\mathbf{x}_{J} \mathbf{y}=\mathbf{c c}_{1}}\right), \psi\left(\left.g\right|_{\mathbf{x}_{J} \mathbf{y}=\mathbf{d c}_{2}}\right)\right)(\tau) .
\end{aligned}
$$

Lemma 2 ( [12, Th. 12]): Let $f$ is a second-order GBF and $G\left(\left.f\right|_{\mathbf{x}_{J}=\mathbf{c}}\right)$ is a path with $x_{\gamma}$ as it's one of the end vertices for all $\mathbf{c} \in\{0,1\}^{k}$. Assume all the edges in the path have the identical weights of $q / 2$. Then for any choice of $g_{i}, g^{\prime} \in \mathbb{Z}_{q}$

$$
\left\{f+\frac{q}{2}\left(\sum_{\alpha=0}^{k-1} u_{\alpha} x_{j_{\alpha}}+u x_{\gamma}\right): u_{\alpha}, u \in\{0,1\}\right\}
$$

is a $\mathrm{CC}$ of size $2^{k+1}$.

Lemma 3 ( [1]): (Construction of CCC)

Let $f$ is a second-order GBF which has the same property as defined in Lemma 2. Consider $\left(t_{0}, t_{1}, \ldots, t_{k-1}\right)$ is the binary representation of the integer $t$. Define the $\mathrm{CC} C_{t}$ to be

$$
\left\{f+\frac{q}{2}\left(\sum_{\alpha=0}^{k-1} u_{\alpha} x_{j_{\alpha}}+\sum_{\alpha=0}^{k-1} t_{\alpha} x_{j_{\alpha}}+u x_{\gamma}\right): u, u_{\alpha} \in\{0,1\}\right\},
$$

and $\bar{C}_{2^{k}+t}$ to be

$$
\left\{\tilde{f}+\frac{q}{2}\left(\sum_{\alpha=0}^{k-1} u_{\alpha} \bar{x}_{j_{\alpha}}+\sum_{\alpha=0}^{k-1} t_{\alpha} \bar{x}_{j_{\alpha}}+\bar{u} x_{\gamma}\right): u, u_{\alpha} \in\{0,1\}\right\} .
$$

Then

$$
\left\{\psi\left(C_{t}\right): 0 \leq t<2^{k}\right\} \cup\left\{\psi^{*}\left(\bar{C}_{2^{k}+t}\right): 0 \leq t<2^{k}\right\}
$$

generate a set of $\mathrm{CCC}$, where $\psi^{*}(\cdot)$ is the complex conjugate of $\psi(\cdot)$.

Before presenting the next lemmas, define $I_{t}=\{0,1, \ldots, t-$ $1\}$. Therefore, $I_{m}$ is a set of indices of the variables 
$x_{0}, x_{1}, \ldots, x_{m-1}$. We assume $J^{\prime}:\left\{j_{0}^{\prime}, j_{1}^{\prime}, \ldots, j_{m-k-1}^{\prime}\right\} \subset$ $I_{m} \backslash J$, and

$$
\begin{cases}W: & \left\{w_{0}, w_{1}, \ldots, w_{k-1}\right\} \subset I_{n},\left\{w_{k}\right\} \subset I_{n} \backslash W \\ W^{\prime}: & \left\{w_{0}^{\prime}, w_{1}^{\prime}, \ldots, w_{n-k-2}^{\prime}\right\} \subset I_{n} \backslash\left\{W, w_{k}\right\} .\end{cases}
$$

The above defined sets will be used for representation of below binary vectors: $\mathbf{x}=\left(x_{0}, x_{1}, \ldots, x_{m-1}\right), \mathbf{u}=$ $\left(u_{0}, u_{1}, \ldots, u_{n-1}\right), \mathbf{u}_{W}=\left(u_{w_{0}}, u_{w_{1}}, \ldots, u_{w_{k-1}}\right)$, and $\mathbf{u}_{W^{\prime}}=$ $\left(u_{w_{0}^{\prime}}, u_{w_{1}^{\prime}}, \ldots, u_{w_{n-k-2}^{\prime}}\right)$.

\section{Lemma 4 ( [3]): (Construction of ZCCS)}

Let $f$ is a second-order GBF. Assume, $G\left(\left.f\right|_{\mathbf{x}_{J}=\mathbf{c}}\right)$ consists of a path with $x_{\gamma}$ as one of its end vertices and $p$ isolated vertices $x_{m-p}, x_{m-p+1}, \ldots, x_{m-1}$. Let $t_{0}, t_{1}, \ldots, t_{k+p-1}$ be the binary vector representation of $t$ then the ordered set $S_{t}$ is defined as

$$
\begin{array}{r}
\left\{f+\frac{q}{2}\left(\sum_{\alpha=0}^{k-1} u_{\alpha} x_{j_{\alpha}}+\sum_{\alpha=0}^{k-1} t_{\alpha} x_{j_{\alpha}}+\sum_{\alpha=0}^{p-1} t_{k+\alpha} x_{m-p+\alpha}\right.\right. \\
\left.\left.+u_{k} x_{\gamma}\right): u_{k}, u_{\alpha} \in \mathbb{Z}_{2}\right\},
\end{array}
$$

and the counterpart set $\bar{S}_{t}$ to be

$$
\begin{gathered}
\left\{\tilde{f}+\frac{q}{2}\left(\sum_{\alpha=0}^{k-1} u_{\alpha} \bar{x}_{j_{\alpha}}+\sum_{\alpha=0}^{k-1} t_{\alpha} \bar{x}_{j_{\alpha}}+\sum_{\alpha=0}^{p-1} t_{k+\alpha} \bar{x}_{m-p+\alpha}\right.\right. \\
\left.\left.+\overline{u_{k}} x_{\gamma}\right): u_{k}, u_{\alpha} \in\{0,1\}\right\} .
\end{gathered}
$$

Then

$\left\{\psi\left(S_{t}\right): 0 \leq t \leq 2^{k+p}-1\right\} \cup\left\{\psi^{*}\left(\bar{S}_{t}\right): 0 \leq t \leq 2^{k+p}-1\right\}$, form $\left(2^{k+p+1}, 2^{m-p}\right)-\mathrm{ZCCS}_{2^{k+1}}^{2^{m}}$.

Lemma 5 ( [15]): For any $(K, Z)-\mathrm{ZCCS}_{M}^{L}$, the theoretical bound is given by

$$
K \leq M\lfloor L / Z\rfloor,
$$

We call $(K, Z)-\mathrm{ZCCS}_{M}^{L}$ is optimal if $K=M\lfloor L / Z\rfloor$.

\section{Proposed New Construction of ZCCS With MaXimum Column SEQuence PMEPR 2}

Before presenting our propose construction, we first define the following notations:

$$
\begin{cases}\mathcal{Z}: \quad & \{m-p, m-p+1, \ldots, m-1\} \subset I_{m} \\ & \text { where } 0 \leq p \leq m-k-1 \\ J_{1}: \quad\left\{j_{0}, j_{1}, \ldots, j_{k-1}\right\} \subset I_{m} \backslash \mathcal{Z} \\ J_{1}^{\prime}: \quad\left\{j_{0}^{\prime}, j_{1}^{\prime}, \ldots, j_{m-k-p-1}^{\prime}\right\} \subset I_{m} \backslash J_{1} \cup \mathcal{Z} .\end{cases}
$$

Here, $J_{1}$ is equals to $J$, when $\mathcal{Z}=\phi$. We also define the following binary vectors: $\mathbf{x}_{\mathcal{Z}}=\left(x_{m-p}, x_{m-p+1}, \ldots, x_{m-1}\right)$ and $\mathbf{x}_{J_{1}}=\left(x_{j_{0}}, x_{j_{1}}, \ldots, x_{j_{k-1}}\right)$. Now, we present a lemma which will be used in our propose construction.

Lemma 6: Let $f$ and $f^{\prime}$ are two GBFs and $\left.f^{\prime}\right|_{\mathbf{x}_{J_{1}}=\mathbf{c}}$ are given by

$$
\begin{aligned}
\left.f\right|_{\mathbf{x}_{J_{1}}=\mathbf{c}}=P+L+g_{m-p} x_{m-p}+g_{m-p+1} x_{m-p+1} & \\
& +\cdots+g_{m-1} x_{m-1}+g^{\prime}, \\
\left.f^{\prime}\right|_{\mathbf{x}_{J_{1}}=\mathbf{c}}=\left.f\right|_{\mathbf{x}_{J_{1}}=\mathbf{c}}+\frac{q}{2} x_{\gamma_{\mathbf{c}}}, &
\end{aligned}
$$

where

$$
\begin{aligned}
P & =\frac{q}{2} \sum_{\alpha=0}^{m-k-p-2} x_{j_{\alpha}^{\prime}} x_{j_{\alpha+1}^{\prime}}, \\
L & =\sum_{\alpha=0}^{m-k-p-1} g_{j_{\alpha}^{\prime}} x_{j_{\alpha}^{\prime}},
\end{aligned}
$$

$g^{\prime}, g_{j_{\alpha}^{\prime}} \in \mathbb{Z}_{q}, \alpha=0,1, \ldots, m-k-p-1$ and $\gamma_{\mathbf{c}}$ is one of the end vertices of the path $G(P)$. Then for fixed $\mathbf{c} \in\{0,1\}^{k}$ and $\mathbf{d}^{\prime} \neq \mathbf{d}^{\prime \prime}$, where $\mathbf{d}^{\prime}=\left(d_{1}^{\prime}, d_{2}^{\prime}, \ldots, d_{p}^{\prime}\right)\left(\in\{0,1\}^{p}\right)$ and $\mathbf{d}^{\prime \prime}=\left(d_{1}^{\prime \prime}, d_{2}^{\prime \prime}, \ldots, d_{p}^{\prime \prime}\right)\left(\{0,1\}^{p}\right)$, we have

$\mathscr{C}\left(\left.f\right|_{\mathbf{x} \mathbf{x}^{\prime}=\mathbf{c d}^{\prime}},\left.f\right|_{\mathbf{x} \mathbf{x}^{\prime}=\mathbf{c d}^{\prime \prime}}\right)(\tau)+\mathscr{C}\left(\left.f^{\prime}\right|_{\mathbf{x} \mathbf{x}^{\prime}=\mathbf{c d}^{\prime}},\left.f^{\prime}\right|_{\mathbf{x} \mathbf{x}^{\prime}=\mathbf{c d}^{\prime \prime}}\right)(\tau)$

$$
=\left\{\begin{array}{c}
\omega^{\left(d_{1}^{\prime}-d_{1}^{\prime \prime}\right) g_{m-p}}+\cdots+\left(d_{p}^{\prime}-d_{p}^{\prime \prime}\right) g_{m-1} 2^{m-(k+p)+1}, \\
\tau=\left(d_{1}^{\prime}-d_{1}^{\prime \prime}\right) 2^{m-p}+\cdots+\left(d_{p}^{\prime}-d_{p}^{\prime \prime}\right) 2^{m-1}, \\
0, \quad \text { otherwise. }
\end{array}\right.
$$

Theorem 1: Let $f\left(\mathbf{x}, \mathbf{u}_{W^{\prime}}\right):\{0,1\}^{n+m-k-1} \rightarrow \mathbb{Z}_{q}$ and $h(\mathbf{u}):\{0,1\}^{n} \rightarrow \mathbb{Z}_{q}$ are two GBFs of of degree greater than 1. Suppose, $f$ has the property that $G\left(\left.f\right|_{\mathbf{x}_{J_{1}}=\mathbf{c}, \mathbf{u}_{W^{\prime}}=\mathbf{e}}\right)$, where $\mathbf{c} \in\{0,1\}^{k}$ and $\mathbf{e} \in\{0,1\}^{n-k-1}$, contains a Hamiltonian path whose vertices are specified by $J_{1}^{\prime}$ and $\gamma_{\mathbf{c}}$ is one of the end vertices. $p$ is the number of isolated vertices which are specified by $\mathcal{Z}$ and the edges in the path are having identical weights $q / 2$. Also, let $\left(t_{0}, t_{1}, \ldots, t_{n+p-2}\right)$ be binary representation of the integer $t$. Define, the code $S_{t}$ to be

$$
\begin{aligned}
\{g+ & \frac{q}{2}\left(\sum_{\alpha=0}^{k-1} u_{w_{\alpha}} x_{j_{\alpha}}+u_{w_{k}} x_{\gamma_{\mathbf{c}}}\right)+\frac{q}{2}\left(\sum_{\alpha=0}^{k-1} t_{\alpha} x_{j_{\alpha}}\right. \\
& \left.\left.+\sum_{\alpha=0}^{n-k-2} t_{\alpha+k} u_{w_{\alpha}^{\prime}}+\sum_{\alpha=0}^{p-1} t_{\alpha+n-1} x_{m-p+\alpha}\right)\right\},
\end{aligned}
$$

and the counterpart code $\bar{S}_{t}$ is defined as

$$
\begin{aligned}
\{\tilde{g}+ & \frac{q}{2}\left(\sum_{\alpha=0}^{k-1} u_{w_{\alpha}} \bar{x}_{j_{\alpha}}+\bar{u}_{w_{k}} x_{\gamma_{\mathbf{c}}}\right)+\frac{q}{2}\left(\sum_{\alpha=0}^{k-1} t_{\alpha} \bar{x}_{j_{\alpha}}\right. \\
& \left.\left.+\sum_{\alpha=0}^{n-k-2} t_{\alpha+k} u_{w_{\alpha}^{\prime}}+\sum_{\alpha=0}^{p-1} t_{\alpha+n-1} \bar{x}_{m-p+\alpha}\right)\right\} .
\end{aligned}
$$

Then

$$
\left\{\psi\left(S_{t}\right): 0 \leq t \leq 2^{n+p-1}-1\right\} \cup\left\{\psi^{*}\left(\bar{S}_{t}\right): 0 \leq t \leq 2^{n+p-1}-1\right\},
$$

form $\left(2^{n+p}, 2^{m-p}\right)-\mathrm{ZCCS}_{2^{n}}^{2^{m}}$ if

$$
\left.h\right|_{\mathbf{u}_{W^{\prime}}, \mathbf{u}_{W} u_{w_{k}}=\mathbf{e}, \mathbf{b} b} \in\left\{\delta, \frac{q}{2}+\delta\right\},
$$

where $\delta \in \mathbb{Z}_{q}$ and $b \mathbf{b} \in\{0,1\}^{k+1}$.

Proof: Please see Appendix A.

Remark 1 (Construction of GBFs as Defined in Theorem 1): The GBF corresponding to Theorem 1 is given by 
$g(\mathbf{x}, \mathbf{u})=f\left(\mathbf{x}, \mathbf{u}_{W^{\prime}}\right)+h(\mathbf{u})$, where the function $f\left(\mathbf{x}, \mathbf{u}_{W^{\prime}}\right)$ can be expressed as

$$
\begin{aligned}
& f\left(\mathbf{x}, \mathbf{u}_{W^{\prime}}\right)= \\
& \frac{q}{2} \sum_{\mathbf{c} \in\{0,1\}^{k}}^{m-k-p-2} \sum_{\alpha=0}^{m} x_{j_{\pi_{\mathbf{c}}(\alpha)}^{\prime}} x_{j_{\pi_{\mathbf{c}}(\alpha+1)}^{\prime}} \prod_{\alpha=0}^{k-1} x_{j_{\alpha}}^{c_{\alpha}}\left(1-x_{j_{\alpha}}\right)^{1-c_{\alpha}} \\
& +\sum_{r=1}^{k} \sum_{0 \leq \alpha_{0}<\alpha_{1}<\cdots<\alpha_{r}<k} \varrho_{j_{\alpha_{0}, j_{\alpha_{1}}, \ldots, j_{\alpha_{r}}}} x_{j_{\alpha_{0}}} x_{j_{\alpha_{1}} \cdots} x_{j_{\alpha_{r}}} \\
& +\sum_{r=1}^{k} \sum_{\alpha=0}^{m-k-p-1} \sum_{0 \leq \alpha_{0}<\alpha_{1}<\cdots<\alpha_{r}<k} \kappa_{j_{\alpha_{0}}, j_{\alpha_{1}}, \ldots, j_{\alpha_{r}}}^{\alpha} x_{j_{\alpha_{0}}} x_{j_{\alpha_{1}} \cdots x_{j_{\alpha_{r}}}} x_{j_{\alpha}^{\prime}} \\
& +\sum_{r=1}^{k} \sum_{\alpha=0}^{p-1} \sum_{0 \leq \alpha_{0}<\alpha_{1}<\cdots<\alpha_{r}<k} \varrho_{j_{\alpha_{0}}, j_{\alpha_{1}}, \ldots, j_{\alpha_{r}}}^{m-p+\alpha} x_{j_{\alpha_{0}}} x_{j_{\alpha_{1}}} \cdots x_{j_{\alpha_{r}}} x_{m-p+\alpha} \\
& +\sum_{r=1}^{k} \sum_{\alpha=0}^{n-k-2} \sum_{0 \leq \alpha_{0}<\alpha_{1}<\cdots<\alpha_{r}<k} \varrho_{j_{\alpha_{0}}, j_{\alpha_{1}}, \ldots, j_{\alpha_{r}}}^{\alpha} x_{j_{\alpha_{0}}} x_{j_{\alpha_{1}}} \cdots x_{j_{\alpha_{r}}} x_{u_{w_{\alpha}^{\prime}}} \\
& +\sum_{\alpha=0}^{m-1} x_{\alpha} \sum_{\beta=0}^{n-k-2} \vartheta_{\alpha}^{\beta} x_{u_{w_{\beta}^{\prime}}}+\sum_{\alpha=0}^{m-1} g_{\alpha} x_{\alpha}+g^{\prime}
\end{aligned}
$$

where

- $\pi_{\mathbf{c}}\left(\mathbf{c} \in\{0,1\}^{k}\right)$ is a permutation on the set $\{0,1, \ldots, m-$ $k-p-1\}$,

- $\varrho_{j_{\alpha_{0}}, j_{\alpha_{1}}, \ldots, j_{\alpha_{r}}} \in \mathbb{Z}_{q}$ for $r=1,2, \ldots, k, 0 \leq \alpha_{0}<\alpha_{1}<$ $\cdots<\alpha_{r}<k$

- $\kappa_{j_{\alpha_{0}}, j_{\alpha_{1}}, \ldots, j_{\alpha_{r}}}^{\alpha} \in \mathbb{Z}_{q}$, for $r=1,2, \ldots, k, 0 \leq \alpha_{0}<\alpha_{1}<$ $\cdots<\alpha_{r}<k, \alpha=0,1, \ldots, m-k-p-1$,

- $\varrho_{j_{\alpha_{0}}, j_{\alpha_{1}}, \ldots, j_{\alpha_{r}}}^{m-p+\alpha} \in \mathbb{Z}_{q}$ for $r=1,2, \ldots, k, 0 \leq \alpha_{0}<\alpha_{1}<$ $\cdots<\alpha_{r}<k, \alpha=0,1, \ldots, p-1$,

- $\varrho_{j_{\alpha_{0}}, j_{\alpha_{1}}, \ldots, j_{\alpha_{r}}}^{\alpha \alpha} \in \mathbb{Z}_{q}$ for $r=1,2, \ldots, k, 0 \leq \alpha_{0}<\alpha_{1}<$ $\cdots<\alpha_{r}<k$

- $\vartheta_{\alpha}^{\beta} \in \mathbb{Z}_{q}, \alpha=0,1, \ldots, m-1, \beta=0,1, \ldots, n-k-2$, $g_{\alpha} \in \mathbb{Z}_{q}, g \in \mathbb{Z}_{q}$,

and the term

$$
\sum_{r=1}^{k} \sum_{\alpha=0}^{p-1} \sum_{0 \leq \alpha_{0}<\alpha_{1}<\cdots<\alpha_{r}<k} \varrho_{j_{0}, j_{1}, \ldots, j_{r}}^{m-p+\alpha} x_{j_{\alpha_{0}}} x_{j_{\alpha_{1}}} \cdots x_{j_{\alpha_{r}}} x_{m-p+\alpha},
$$

is denoted by $\mathcal{G}_{\mathbf{x}_{J_{1}} \mathbf{x}_{\mathcal{Z}}}$ in the proof of Theorem 1 and also will be used in the construction of IGC code set. The function $h(\mathbf{u})$ can be taken to be of any order. For our desired result (as we are interested to design ZCCS of maximum column sequence PMEPR 2) we take the function as follows:

$$
h(\mathbf{u})=\frac{q}{2} \sum_{\alpha=0}^{n-2} u_{\pi(\alpha)} u_{\pi(\alpha+1)}+\sum_{\alpha=0}^{n-1} \lambda_{\alpha} u_{\alpha}+\lambda^{\prime},
$$

where $\pi$ is a permutation on the set $\{0,1, \ldots, n-1\}, \lambda_{\alpha} \in \mathbb{Z}_{q}$ for $\alpha=0,1, \ldots, n-1$, and $\lambda^{\prime} \in \mathbb{Z}_{q}$.

It is noted that we design $f$ such a way that $G\left(\left.f\right|_{\mathbf{x}=\mathbf{c}}\right)$ contains path over the vertices $x_{j_{\pi_{\mathbf{c}}(0)}^{\prime}}, x_{j_{\pi_{\mathbf{c}}(1)}^{\prime}}, \ldots, x_{j_{\pi_{\mathbf{c}}(m-k-p-1)}^{\prime}}$ and $p$ isolated vertices $x_{m-p}, x_{m-p+1}, \ldots, x_{m-1}$. It is also noted that $x_{j_{\pi_{\mathbf{c}}(0)}^{\prime}}$ and $x_{j_{\pi_{\mathbf{c}}(m-k-p-1)}^{\prime}}$ are the end vertices in the path which is contained in $G\left(\left.f\right|_{\mathbf{x}=\mathbf{c}}\right)$ and in Theorem $1, \gamma_{\mathbf{c}}$ is taken as either $j_{\pi_{\mathbf{c}}(0)}^{\prime}$ or $j_{\pi_{\mathbf{c}}(m-k-p-1)}^{\prime}$.
Corollary 1: From Theorem 1, we obtain $K\left(=2^{n+p}\right)$ codes where each code contains $M\left(=2^{n}\right)$ constituent sequences of length $L\left(=2^{m}\right)$ with ZCZ width $Z\left(=2^{m-p}\right)$. The code set also satisfies the equality $K=\frac{L M}{Z}$ and thus, $\left(2^{n+p}, 2^{m-p}\right)$ $\mathrm{ZCCS}_{2^{n}}^{2^{m}}$ is an optimal ZCCS.

Corollary 2: In Theorem 1, we take $f\left(\mathbf{x}, \mathbf{u}_{W^{\prime}}\right)$ and $h(\mathbf{u}$ as given in (26) and (28) respectively. As, $g(\mathbf{x}, \mathbf{u})=$ $f\left(\mathbf{x}, \mathbf{u}_{W^{\prime}}\right)+h(\mathbf{u}$ and $G(g(\mathbf{c}, \mathbf{u}))$ is a path over the vertices $u_{0}, u_{1}, \ldots, u_{n-1}$ for all $\mathbf{c} \in\{0,1\}^{m}$, the maximum column sequence PMEPR of the $\left(2^{n+p}, 2^{m-p}\right)-\mathrm{ZCCS}_{2^{n}}^{2^{m}}$ is 2 .

Proof: We recall the set $S_{t}$ given in (22) and assume

$$
\begin{aligned}
\mathcal{F}_{\mathbf{x}, \mathbf{u}}^{t}=g & +\frac{q}{2}\left(\sum_{\alpha=0}^{k-1} u_{w_{\alpha}} x_{j_{\alpha}}+u_{w_{k}} x_{\gamma}\right) \\
+ & \frac{q}{2}\left(\sum_{\alpha=0}^{k-1} t_{\alpha} x_{j_{\alpha}}+\sum_{\alpha=0}^{n-k-2} t_{\alpha+k} u_{w_{\alpha}^{\prime}}\right. \\
& \left.+\sum_{\alpha=0}^{p-1} t_{\alpha+n-1} x_{m-p+\alpha}\right)
\end{aligned}
$$

Let $\mathbf{x}_{0}, \mathbf{x}_{1}, \ldots, \mathbf{x}_{m-1}$ are the binary vector representations of $0,1, \ldots, 2^{m}-1$, and $\mathbf{u}_{0}, \mathbf{u}_{1}, \ldots, \mathbf{u}_{n-1}$ are the binary vector representations of $0,1, \ldots, 2^{n}-1$. Therefore,

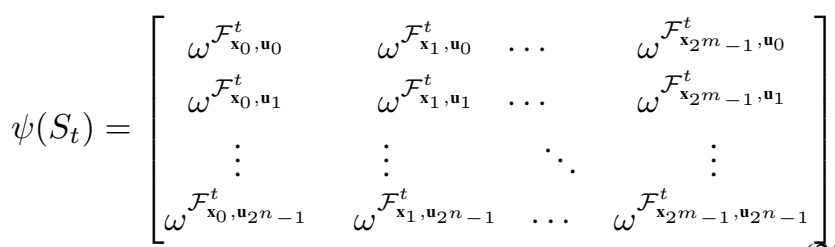

The $j$ th column of the code $\psi\left(S_{t}\right)$ is denoted by $\psi\left(\mathcal{F}_{\mathbf{x}_{j}, \mathbf{u}}^{t}\right)$ and given by $\left[\begin{array}{lllll}\omega^{\mathcal{F}_{\mathbf{x}_{j}, \mathbf{u}_{0}}^{t}} & \omega^{\mathcal{F}_{\mathbf{x}_{j}, \mathbf{u}_{1}}^{t}} & \cdots & \omega^{\mathcal{F}_{\mathbf{x}_{j}, \mathbf{u}_{2} n_{-1}}^{t}}\end{array}\right]^{T}$, where $j=$ $0,1, \ldots, 2^{m}-1$. If we setup $k=0$ in Lemma 2 , i.e., if $G(f)$ is a path, the set $\left\{\psi(f), \psi\left(f+\frac{q}{2} x_{\gamma}\right)\right\}$ forms a GCP. From (29), it is clear that $G\left(\mathcal{F}_{\mathbf{x}_{j}, \mathbf{u}}^{t}\right)$ is the same as $G\left(g\left(\mathbf{x}_{j}, \mathbf{u}\right)\right)$ which is a path for all $j$ over the vertices $u_{0}, u_{1}, \ldots, u_{n-1}$. Therefore, by using Lemma 2, the set $\left\{\psi\left(\mathcal{F}_{\mathbf{x}_{j}, \mathbf{u}}^{t}\right), \psi\left(\mathcal{F}_{\mathbf{x}_{j}, \mathbf{u}}^{t}+u_{\gamma}\right)\right\}$ forms a GCP where $u_{\gamma}$ is assumed to be one of the end vertices of the path $G\left(\mathcal{F}_{\mathbf{x}_{j}, \mathbf{u}}^{t}\right)$. Therefore, each column of the code $\psi\left(S_{t}\right)$ lies in a GCP and hence the maximum PMEPR is 2. Similarly, we can show that the maximum PMEPR of each column of the code $\psi^{*}\left(\bar{S}_{t}\right)$ is 2 . Therefore, the maximum column sequence PMEPR of the ZCCS $\left\{\psi\left(S_{t}\right): 0 \leq t \leq 2^{n+p-1}-1\right\} \cup$ $\left\{\psi^{*}\left(\bar{S}_{t}\right): 0 \leq t \leq 2^{n+p-1}-1\right\}$ or, $\left(2^{n+p}, 2^{m-p}\right)-\mathrm{ZCCS}_{2^{n}}^{2^{m}}$ is 2.

Remark 2: For the case, $\mathcal{Z}=\phi$, the result of Theorem 1 reduces to the result given in [14]. Therefore, the construction given in [14] appears as special case of the proposed ZCCS construction.

Remark 3: For $W \cup W^{\prime} \cup\left\{w_{k}\right\}=\phi$, the function $g$ given in Theorem 1 reduces to $g=f$. If we consider the degree of $f$ is 2 and $W \cup W^{\prime} \cup\left\{w_{k}\right\}=\phi$, the result of Theorem 1 reduces to the result given in [3, Th. 2]. Therefore, the construction given in [3] and [12], which appear as Lemma 2 and Lemma 4 repectively in this paper, are special cases of our proposed construction.

Below, we present an example to illustrate Theorem 1. 
TABLE I: Optimal ZCCS over the alphabet $\mathbb{Z}_{4}$ with maximum column sequence PMEPR 2.

\begin{tabular}{|c|c|}
\hline \multicolumn{2}{|c|}{$(8,16)-\mathrm{ZCC}_{4}^{32}$} \\
\hline$\psi\left(S_{0}\right)$ & $\psi\left(S_{1}\right)$ \\
\hline 22330031001300333102130013221302 & 20310233021102313300110211201100 \\
20310233021102313300110211201100 & 22330031001300333102130013221302 \\
22110013003100113120132213001320 & 20130211023302133322112011021122 \\
02312033201120311100330233203300 & 00332231221322331302310031223102 \\
\hline$\psi\left(S_{2}\right)$ & $\psi\left(S_{3}\right)$ \\
\hline 22330031001300331320312231003120 & 20310233021102311122332033023322 \\
20310233021102311122332033023322 & 22330031001300331320312231003120 \\
22110013003100111302310031223102 & 20130211023302131100330233203300 \\
02312033201120313322112011021122 & 00332231221322333120132213001320 \\
\hline$\psi^{*}\left(S_{0}\right)$ & $\psi^{*}\left(S_{1}\right)$ \\
\hline 20312231003120131122132231221100 & 00110211201100333102330211023120 \\
00110211201100333102330211023120 & 20312231003120131122132231221100 \\
20132213001320311100130031001122 & 00330233203300113120332011203102 \\
22112011021122331302110233021320 & 02310031223102133322312213223300 \\
\hline$\psi^{*}\left(\bar{S}_{3}\right)$ & $\psi^{*}\left(\bar{S}_{4}\right)$ \\
\hline 02130013221302311122132231221100 & 22332033023322113102330211023120 \\
22332033023322113102330211023120 & 02130013221302311122132231221100 \\
02310031223102131100130031001122 & 22112011021122333120332011203102 \\
00330233203300111302110233021320 & 20132213001320313322312213223300 \\
\hline
\end{tabular}
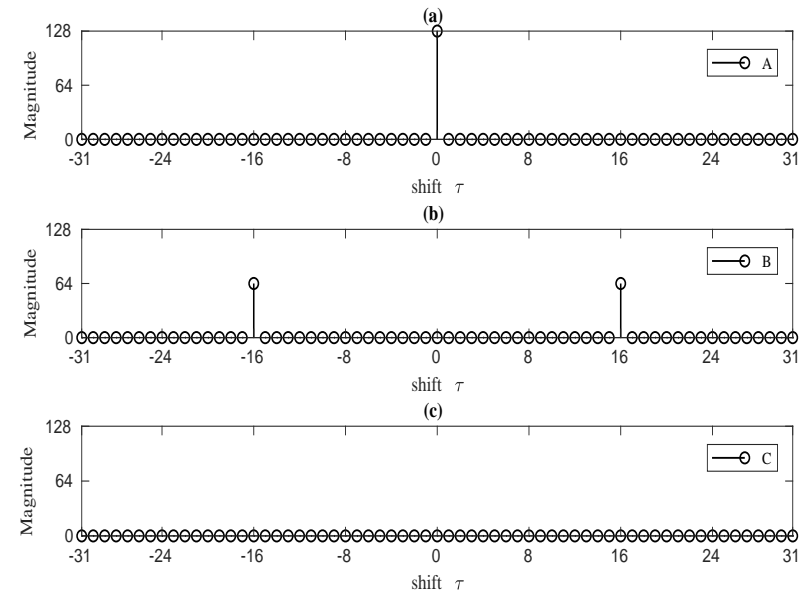

Fig. 1: Correlation plots of $(8,16)-\mathrm{ZCCS}_{4}^{32}$ in TABLE I.

Example 1: We consider $m=5, n=2, k=1, p=1, W=$ $\left\{w_{0}\right\}=\{0\}$, and $\left\{w_{1}\right\}=\{1\}$. Therefore, $W^{\prime}$ is a null set. We also consider Let $f:\{0,1\}^{5} \rightarrow \mathbb{Z}_{4}$ be a GBF given by

$$
\begin{aligned}
f= & 2\left(\left(1-x_{0}\right)\left(x_{3} x_{2}+x_{2} x_{1}\right)+x_{0}\left(x_{1} x_{3}+x_{3} x_{2}\right)+x_{0} x_{4}\right. \\
& +x_{1}+2 x_{2}+2 x_{3}+x_{4}+2,
\end{aligned}
$$

and the function $h\left(u_{0}, u_{1}\right)$ is given by

$$
h\left(u_{0}, u_{1}\right)=2 u_{0} u_{1} .
$$

From $f$, it is clear that $J_{1}=\{0\}, J_{1}^{\prime}=\{1,2,3\}$, and $\mathcal{Z}=$ $\{4\}$. Therefore, from Theorem 1 and Corollary 2, we obtain $(8,16)-\mathrm{ZCCS}_{4}^{32}$ with maximum column sequence PMEPR 2. The $(8,16)-\mathrm{ZCCS}_{4}^{32}$ is given in TABLE I. In TABLE I, $\psi\left(S_{t}\right)$ and $\psi^{*}\left(\bar{S}_{t}\right)$ are obtained by following (22) and (23). In Fig.
1, Fig. 1-(a) represents AACF of any codes given in TABLE I, and Fig. 1-(b) and Fig. 1-(c) represent the ACCFs between two distinct codes in TABLE I.

\section{Proposed New Construction of IGC Code Set With MaXimum Column Sequence PMEPR 2}

In this section, a new construction of IGC code set has been presented by using GBFs of order no less than 2 . We recall $\left(t_{0}, t_{1}, \ldots, t_{n+p-2}\right)$ be binary representation of the integer $t\left(0 \leq t \leq 2^{n+p-1}-1\right)$, where $\mathbf{t}_{1}=\left(t_{0}, t_{1}, \ldots, t_{k-1}\right), \mathbf{t}_{2}=\left(t_{k}, t_{k+1}, \ldots, t_{n-2}\right)$, and $\mathbf{t}_{3}=$ $\left(t_{n-1}, t_{n}, \ldots, t_{n+p-2}\right)$. We assume $\left(t_{0}, t_{1}, \ldots, t_{n-2}\right)$, or, $\mathbf{t}_{1} \mathbf{t}_{2}$ be the binary vector representation of $\mathcal{T}\left(0 \leq \mathcal{T} \leq 2^{n-1}-1\right)$. Also $\left(t_{0}, t_{1}, \ldots, t_{n+p-2}\right)$ and $\mathbf{t}_{1} \mathbf{t}_{2} \mathbf{t}_{3}$ represent the same binary vectors. Now, we define the following code groups $\mathcal{I}_{0}, \mathcal{I}_{1}, \ldots, \mathcal{I}_{2^{n-1}-1}, \overline{\mathcal{I}}_{0}^{*}, \overline{\mathcal{I}}_{1}^{*}, \ldots, \overline{\mathcal{I}}_{2^{n-1}-1}^{*}$ as follows:

$$
\mathcal{I}_{\mathcal{T}}=\left\{\psi\left(S_{t}\right): \mathbf{t}_{3} \in\{0,1\}^{p}\right\},
$$

and

$$
\overline{\mathcal{I}}_{\mathcal{T}}^{*}=\left\{\psi^{*}\left(\bar{S}_{t}\right): \mathbf{t}_{3} \in\{0,1\}^{p}\right\},
$$

where $0 \leq \mathcal{T} \leq 2^{n-1}-1$.

Theorem 2: Let $f\left(\mathbf{x}, \mathbf{u}_{W^{\prime}}\right):\{0,1\}^{n+m-k-1} \rightarrow \mathbb{Z}_{q}$ and $h(\mathbf{u}):\{0,1\}^{n} \rightarrow \mathbb{Z}_{q}$ be two $q$-ary GBFs as defined in (26) and (28) respectively. We also assume $\mathcal{G}_{\mathbf{x}_{J_{1}} \mathbf{x}_{\mathcal{Z}}}=0$. Then the code groups $\mathcal{I}_{0}, \mathcal{I}_{1}, \ldots, \mathcal{I}_{2^{n-1}-1}, \overline{\mathcal{I}}_{0}^{*}, \overline{\mathcal{I}}_{1}^{*}, \ldots, \overline{\mathcal{I}}_{2^{n-1}-1}^{*}$ form an IGC code set $\mathcal{I}\left(2^{n+p}, 2^{n}, 2^{m}, 2^{m-p}\right)$.

Proof: Please see Appendix B.

Corollary 3: In Theorem 2, $g(\mathbf{x}, \mathbf{u})=f\left(\mathbf{x}, \mathbf{u}_{W^{\prime}}\right)+h(\mathbf{u})$, where $f\left(\mathbf{x}, \mathbf{u}_{W^{\prime}}\right)$ is given in (26) with $\mathcal{G}_{\mathbf{x}_{J_{1}} \mathbf{x} \mathcal{Z}}=0$ and $h(\mathbf{u})$ is given in (28). Therefore, $G(g(\mathbf{c}, \mathbf{u}))$ is a path over the vertices $u_{0}, u_{1}, \ldots, u_{n-1}$ for all $\mathbf{c} \in\{0,1\}^{m}$. Hence, by following the 
TABLE II: $\mathcal{I}(8,4,32,16)$ over the alphabet $\mathbb{Z}_{4}$ with maximum column sequence PMEPR 2.

\begin{tabular}{|c|c|c|c|}
\hline \multicolumn{4}{|l|}{$\mathcal{I}_{0}$} \\
\hline \multirow{4}{*}{$\psi\left(S_{0}\right)$} & 23012321123230303012303223030101 & \multirow{4}{*}{$\psi\left(S_{2}\right)$} & 23012321123230301230121001212323 \\
\hline & 21032123103032323210323021010303 & & 21032123103032321032101203232121 \\
\hline & 23232303121030123030301023210123 & & 23232303121030121212123201032301 \\
\hline & 03030323323010321010103003012103 & & 03030323323010323232321221230321 \\
\hline \multicolumn{4}{|r|}{ (2) } \\
\hline \multirow{4}{*}{$\psi\left(S_{1}\right)$} & 21032123103032323210323021010303 & \multirow{4}{*}{$\psi\left(S_{3}\right)$} & 21032123103032321032101203232121 \\
\hline & 23012321123230303012303223030101 & & 23012321123230301230121001212323 \\
\hline & 21212101101232103232321221230321 & & 21212101101232101010103003012103 \\
\hline & 01010121303212301212123201032301 & & 01010121303212303030301023210123 \\
\hline \multicolumn{4}{|r|}{ 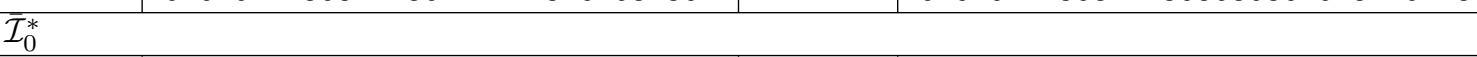 } \\
\hline \multirow{4}{*}{$\left.\psi(\overline{(} S)_{0}\right)$} & 30121030212323230123210132303030 & \multirow{4}{*}{$\psi\left(\overline{(}(S)_{2}\right)$} & 12303212030101010123210132303030 \\
\hline & 10323010010303032103012112101010 & & 32101232232121212103012112101010 \\
\hline & 30301012210123010101212332123012 & & 12123230032301230101212332123012 \\
\hline & 32321210230321030303232130103210 & & 10103032012103210303232130103210 \\
\hline \multicolumn{4}{|c|}{ ( } \\
\hline \multirow{4}{*}{$\psi\left((\overline{(} S)_{1}\right)$} & 10323010010303032103012112101010 & \multirow{4}{*}{$\psi\left(S_{3}\right)$} & 32101232232121212103012112101010 \\
\hline & 30121030212323230123210132303030 & & 12303212030101010123210132303030 \\
\hline & 10103032012103212121010312321032 & & 32321210230321032121010312321032 \\
\hline & 12123230032301232323030110301230 & & 30301012210123012323030110301230 \\
\hline
\end{tabular}

proof of Corollary 2, the maximum column sequence PMEPR of the IGC code set $\mathcal{I}\left(2^{n+p}, 2^{n}, 2^{m}, 2^{m-p}\right)$ is 2 .

We have illustrated Theorem 2 in the below given example.

Example 2: We consider $m=5, n=2, k=1, p=1, W=$ $\left\{w_{0}\right\}=\{0\},\left\{w_{1}\right\}=\{1\}$. Therefore, $W^{\prime}$ is a null set. We also consider Let $f:\{0,1\}^{5} \rightarrow \mathbb{Z}_{4}$ be a GBF given by

$$
\begin{array}{r}
f=2\left(\left(1-x_{0}\right)\left(x_{3} x_{2}+x_{2} x_{1}\right)+x_{0}\left(x_{1} x_{3}+x_{3} x_{2}\right)\right. \\
+x_{0}+2 x_{1}+3 x_{3}+x_{4}+2,
\end{array}
$$

and the function $h\left(u_{0}, u_{1}\right)$ is given by

$$
h\left(u_{0}, u_{1}\right)=2 u_{0} u_{1} .
$$

From $f$, it is clear that $J_{1}=\{0\}, J_{1}^{\prime}=\{1,2,3\}, \mathcal{Z}=\{4\}$, $\mathbf{x}_{J_{1}}=\left(x_{0}\right), \mathbf{x}_{\mathcal{Z}}=\left(x_{4}\right)$ and $\mathcal{G}_{\mathbf{x}_{J_{1}} \mathbf{x}_{\mathcal{Z}}}=0$. From Theorem 2, Corollary 3, (33), and (34), we obtain $\mathcal{I}(8,4,32,16)$. The code groups $\mathcal{I}_{0}, \mathcal{I}_{1}, \overline{\mathcal{I}}_{0}^{*}, \overline{\mathcal{I}}_{1}^{*}$ are given in TABLE II. Fig. 2-(a)
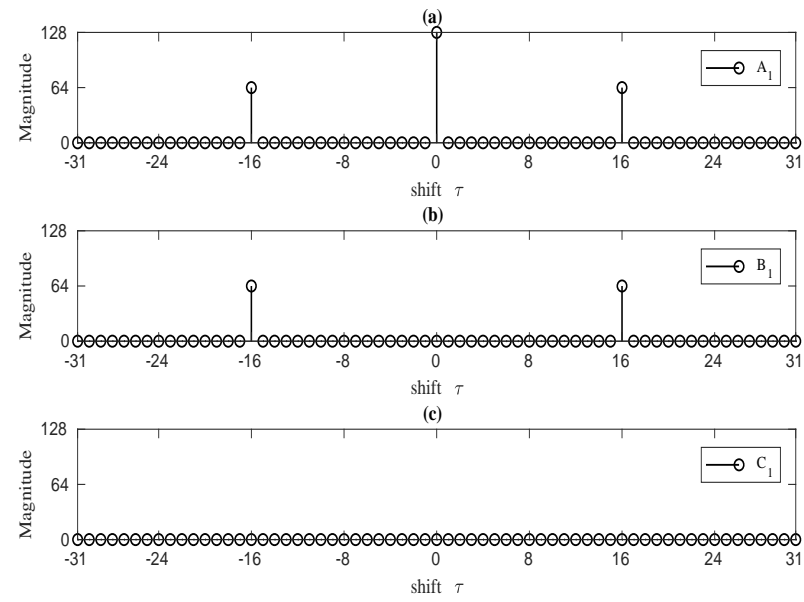

Fig. 2: Correlation plots of $\mathcal{I}(8,4,32,16)$ in TABLE II. represents AACF of any code given in TABLE II. Fig. 2-(b) represents AACF of between two distinct codes from same code group and Fig. 2-(c) represents AACF of between two codes from different code groups in TABLE II.

\section{Comparison of the Proposed Construction With EXISTING ZCCS AND IGC CODE SET CONSTRUCTIONS}

In this section, we compare our proposed ZCCS construction with the construction given in [3]-[5] and the proposed IGC code set construction with [19], [20].

The constructions of ZCCS given in [3] and [4] both are based on second-order GBFs but the maximum column sequence PMEPR depends on the number of subcarriers or the number of sequences in the code. In order to increase number of users in a ZCCS based MC-CDMA system with large ZCZ, we need to increase number of subcarriers which increase column sequence PMEPR. For this scenario all GBFs based ZCCS degrades the performance of a ZCCS based MC-CDMA system. Our proposed constrcution of both ZCCS and IGC code set are based on higher-order $(\geq 2)$ GBFs with maximum column sequence PMEPR 2. Additionaly, we have linked our proposed ZCCS with higher order RM code unlike the order of GBFs used in [3], [4]. Although, the constrcution given in [5] can generate ZCCS with maximum column sequence PMEPR 2 but the constrcution is based on Golay sequences with large zero autocorrelation zone and orthogonal matrix. Therefore, the constrcution given in [5] may not be suitable for fast hardware generation specially for long ZCCS [14].

The constrcution of IGC code set given in [20] is based on second-order Boolean function but maximum column sequence PMEPR depends on the number of constituent sequences in the code. Therefore, in a large subcarrier MCCDMA system, the IGC code set obtained from [20] cannot provide a tight PMEPR upper bound unlike the proposed 
TABLE III: Comparison of proposed construction with [3]-[5], [19], [20]

\begin{tabular}{|c|c|c|c|}
\hline Code Set & Method & PMEPR & Remark \\
\hline ZCCS [3] & $\begin{array}{l}\text { Based on } \\
\text { second-order } \\
\text { GBFs }\end{array}$ & $>2$ & Direct \\
\hline ZCCS [4] & $\begin{array}{l}\text { Based on } \\
\text { second-order } \\
\text { GBFs }\end{array}$ & $>2$ & Direct \\
\hline ZCCS [5] & $\begin{array}{l}\text { Golay sequences } \\
\text { with large zero } \\
\text { autocorrelation } \\
\text { zone and } \\
\text { orthogonal } \\
\text { matrix }\end{array}$ & 2 & Indirect \\
\hline IGC [19] & $\begin{array}{l}\text { Based on } \\
\text { CCCs and } \\
\text { orthogonal } \\
\text { matrix }\end{array}$ & $>2$ & Indirect \\
\hline IGC [20] & $\begin{array}{l}\text { Based on } \\
\text { second-order } \\
\text { GBFs }\end{array}$ & $>2$ & Direct \\
\hline Proposed ZCCS & $\begin{array}{l}\text { Based on } \\
\text { higher-order }(\geq 2) \\
\text { GBFs }\end{array}$ & 2 & Direct \\
\hline Proposed IGC & $\begin{array}{l}\text { Based on } \\
\text { higher-order }(\geq 2) \\
\text { GBFs }\end{array}$ & 2 & Direct \\
\hline
\end{tabular}

IGC code set. The IGC code set construction given in [19] is based on CCCs and orthogonal matrix as well as the maximum column sequence PMEPR depends on the number of constituent sequences in the code. Therefore, the IGC code set obtained from [19] may not be suitable for fast hardware generation as well as for a large subcarrier MC-CDMA system where the high PMEPR value may not be acceptable. Hence, our proposed construction is more suitable than the above mentioned constructions. We also have provided a comparison table for column sequence PMEPR for ZCCS and IGC code set in TABLE III.

\section{CONCLUSION}

This paper focuses on a direct construction of ZCCS and IGC code set with maximum column sequence PMEPR 2. Both the constructions are based on GBFs of order no less than 2. We also have linked our proposed construction with graph. The construction of ZCCS achieves the theoritical upper bound. The maximum column sequence PMEPR of existed ZCCS, based on GBFs depend on number of constituent sequence in a code. Also, the maximum column sequence PMEPR of all existed IGC code sets depend on the number of constituent sequences in a code.

\section{APPENDix A}

\section{PROOF OF Theorem 1}

To prove Theorem 1, it is enough to show that the AACF of any code from the set given in (24) is zero for all nonzero time shifts inside the ZCZ, $2^{m-p}$ and the ACCF of any two codes is zero for all time shifts inside the ZCZ, $2^{m-p}$. We define the following binary vectors: $\mathbf{t}_{1}=\left(t_{0}, t_{1}, \ldots, t_{k-1}\right), \mathbf{t}_{2}=$ $\left(t_{k}, t_{k+1}, \ldots, t_{n-2}\right)$, and $\mathbf{t}_{3}=\left(t_{n-1}, t_{n}, \ldots, t_{n+p-2}\right)$, where $\left(t_{0}, t_{1}, \ldots, t_{n+p-2}\right)$ is the binary representation of the integer $t$ which already define in Theorem 1 . Also, we define $\mathbf{t}_{1}^{\prime}=\left(t_{0}^{\prime}, t_{1}^{\prime}, \ldots, t_{k-1}^{\prime}\right), \mathbf{t}_{2}^{\prime}=\left(t_{k}^{\prime}, t_{k+1}^{\prime}, \ldots, t_{n-2}^{\prime}\right)$, and $\mathbf{t}_{3}^{\prime}=\left(t_{n-1}^{\prime}, t_{n}^{\prime}, \ldots, t_{n+p-2}^{\prime}\right)$, where $\left(t_{0}^{\prime}, t_{1}^{\prime}, \ldots, t_{n+p-2}^{\prime}\right)$ is the binary representation of the integer $t^{\prime}$. In the expression of $S_{t}$ and $\bar{S}_{t}$, we assume

$$
\begin{aligned}
\eta_{1} & =\frac{q}{2}\left(\sum_{\alpha=0}^{k-1} u_{w_{\alpha}} x_{j_{\alpha}}+u_{w_{k}} x_{\gamma}\right) \\
& =\frac{q}{2}\left(\mathbf{u}_{W} \cdot \mathbf{x}_{J_{1}}+u_{w_{k}} x_{\gamma}\right), \\
\eta_{2} & =\frac{q}{2}\left(\sum_{\alpha=0}^{k-1} u_{w_{\alpha}} \bar{x}_{j_{\alpha}}+\bar{u}_{w_{k}} x_{\gamma}\right) \\
& =\frac{q}{2}\left(\mathbf{u}_{W} \cdot \overline{\mathbf{x}}_{J_{1}}+\bar{u}_{w_{k}} x_{\gamma}\right),
\end{aligned}
$$

, where $\overline{\mathbf{x}}_{J_{1}}=\mathbf{1}_{k}-\mathbf{x}_{J_{1}}$ and $\bar{u}_{w_{k}}=1-u_{w_{k}}$. Let us start with $\mathscr{C}\left(\psi\left(S_{t}\right), \psi\left(S_{t^{\prime}}\right)\right)(\tau)$, where the expression denotes the ACCF of the codes $\psi\left(S_{t}\right)$ and $\psi\left(S_{t^{\prime}}\right)$ at the time shift $\tau$ when $t \neq t^{\prime}$, and AACF of $\psi\left(S_{t}\right)$ (or, $\psi\left(S_{t^{\prime}}\right)$ ) at the time shift $\tau$ when $t=t^{\prime}$. The expression $\mathscr{C}\left(\psi\left(S_{t}\right), \psi\left(S_{t^{\prime}}\right)\right)(\tau)$ can be written as

$$
\begin{aligned}
& \mathscr{C}\left(\psi\left(S_{t}\right), \psi\left(S_{t^{\prime}}\right)\right)(\tau) \\
& =\sum_{\mathbf{u} \in\{0,1\}^{n}} \mathscr{C}\left(g+\eta_{1}+\frac{q}{2}\left(\mathbf{t}_{1} \cdot \mathbf{x}_{J_{1}}+\mathbf{t}_{2} \cdot \mathbf{u}_{W^{\prime}}+\mathbf{t}_{3} \cdot \mathbf{x}_{\mathcal{Z}}\right),\right. \\
& =\sum_{\mathbf{u}_{W^{\prime}}=\mathbf{e}}(-1)^{\left(\mathbf{t}_{2}-\mathbf{t}_{2}^{\prime}\right) \cdot \mathbf{e}} \\
& \quad \times\left(\sum _ { \mathbf { u } _ { W } , u _ { w _ { k } } } \mathscr { C } \left(\left.f\right|_{\mathbf{u}_{W^{\prime}}=\mathbf{e}}+\eta_{1}+\frac{q}{2}\left(\mathbf{t}_{1} \cdot \mathbf{x}_{J_{1}}+\mathbf{t}_{3} \cdot \mathbf{x}_{\mathcal{Z}}\right),\right.\right. \\
& \left.\left.\quad f \mathbf{x}_{J_{1}}+\mathbf{t}_{2}^{\prime} \cdot \mathbf{u}_{W^{\prime}}+\mathbf{t}_{3}^{\prime} \cdot \mathbf{x}_{\mathcal{Z}}\right)\right)(\tau) \\
& \left.\left.=\sum_{\mathbf{u}_{W^{\prime}}=\mathbf{e}}+\eta_{1}+\frac{q}{2}\left(\mathbf{t}_{1}^{\prime} \cdot \mathbf{x}_{J_{1}}+\mathbf{t}_{3}^{\prime} \cdot \mathbf{x}_{\mathcal{Z}}\right)\right)(\tau)\right)
\end{aligned}
$$

where

$$
\begin{gathered}
\mathcal{A}=\left(\sum _ { \mathbf { u } _ { W } , u _ { w _ { k } } } \mathscr { C } \left(\left.f\right|_{\mathbf{u}_{W^{\prime}}=\mathbf{e}}+\eta_{1}+\frac{q}{2}\left(\mathbf{t}_{1} \cdot \mathbf{x}_{J_{1}}+\mathbf{t}_{3} \cdot \mathbf{x}_{\mathcal{Z}}\right),\right.\right. \\
\left.\left.\left.f\right|_{\mathbf{u}_{W^{\prime}}=\mathbf{e}}+\eta_{1}+\frac{q}{2}\left(\mathbf{t}_{1}^{\prime} \cdot \mathbf{x}_{J_{1}}+\mathbf{t}_{3}^{\prime} \cdot \mathbf{x}_{\mathcal{Z}}\right)\right)(\tau)\right) .
\end{gathered}
$$

Now, $\mathcal{A}$ given in (39), can be expressed as

$$
\mathcal{A}=\mathcal{A}_{1}+\mathcal{A}_{2},
$$

where

$$
\begin{aligned}
\mathcal{A}_{1}=( & \sum_{\mathbf{u}_{W}, u_{w_{k}}} \sum_{\mathbf{c}_{1} \neq \mathbf{c}_{2}} \mathscr{C}\left(\left.f\right|_{\mathbf{u}_{W^{\prime}}=\mathbf{e}, \mathbf{x}_{J_{1}}=\mathbf{c}_{1}}+\left.\eta_{1}\right|_{\mathbf{x}_{J_{1}}=\mathbf{c}_{1}}\right. \\
& +\frac{q}{2}\left(\mathbf{t}_{1} \cdot \mathbf{c}_{1}+\mathbf{t}_{3} \cdot \mathbf{x}_{\mathcal{Z}}\right),\left.f\right|_{\mathbf{u}_{W^{\prime}}=\mathbf{e}, \mathbf{x}_{J_{1}}=\mathbf{c}_{2}}+\left.\eta_{1}\right|_{\mathbf{x}_{J_{1}}=\mathbf{c}_{2}} \\
& \left.\left.+\frac{q}{2}\left(\mathbf{t}_{1}^{\prime} \cdot \mathbf{c}_{2}+\mathbf{t}_{3}^{\prime} \cdot \mathbf{x}_{\mathcal{Z}}\right)\right)(\tau)\right),
\end{aligned}
$$




$$
\begin{aligned}
\mathcal{A}_{2}= & \sum_{\mathbf{u}_{W}, u_{w_{k}}} \sum_{\mathbf{c} \in\{0,1\}^{k}} \mathscr{C}\left(\left.f\right|_{\mathbf{u}_{W^{\prime}}=\mathbf{e}, \mathbf{x}_{J_{1}}=\mathbf{c}}+\left.\eta_{1}\right|_{\mathbf{x}_{J_{1}}=\mathbf{c}}\right. \\
& +\frac{q}{2}\left(\mathbf{t}_{1} \cdot \mathbf{c}+\mathbf{t}_{3} \cdot \mathbf{x}_{\mathcal{Z}}\right),\left.f\right|_{\mathbf{u}_{W^{\prime}}=\mathbf{e}, \mathbf{x}_{J_{1}}=\mathbf{c}}+\left.\eta_{1}\right|_{\mathbf{x}_{J_{1}}=\mathbf{c}} \\
& \left.\left.+\frac{q}{2}\left(\mathbf{t}_{1}^{\prime} \cdot \mathbf{c}+\mathbf{t}_{3}^{\prime} \cdot \mathbf{x}_{\mathcal{Z}}\right)\right)(\tau)\right) .
\end{aligned}
$$

From (37) and (41), we have

$$
\begin{aligned}
\mathcal{A}_{1}=( & \sum_{u_{w_{k}}} \sum_{\mathbf{c}_{1} \neq \mathbf{c}_{2}} \mathscr{C}\left(\left.f\right|_{\mathbf{u}_{W^{\prime}}=\mathbf{e}, \mathbf{x}_{J_{1}}=\mathbf{c}_{1}}+\frac{q}{2} u_{w_{k}}\right. \\
& +\frac{q}{2}\left(\mathbf{t}_{1} \cdot \mathbf{c}_{1}+\mathbf{t}_{3} \cdot \mathbf{x}_{\mathcal{Z}}\right),\left.f\right|_{\mathbf{u}_{W^{\prime}}=\mathbf{e}, \mathbf{x}_{J_{1}}=\mathbf{c}_{2}}+\frac{q}{2} u_{w_{k}} \\
& \left.\left.+\frac{q}{2}\left(\mathbf{t}_{1}^{\prime} \cdot \mathbf{c}_{2}+\mathbf{t}_{3}^{\prime} \cdot \mathbf{x}_{\mathcal{Z}}\right)\right)(\tau) \sum_{\mathbf{u}_{W}}(-1)^{\mathbf{u}_{W} \cdot\left(\mathbf{c}_{1}-\mathbf{c}_{2}\right)}\right) .
\end{aligned}
$$

Since, $\mathbf{c}_{1} \neq \mathbf{c}_{2}, \sum_{\mathbf{u}_{W}}(-1)^{\mathbf{u}_{W} \cdot\left(\mathbf{c}_{1}-\mathbf{c}_{2}\right)}=0$ and therefore, from (41)

$$
\mathcal{A}_{1}=0 \forall \tau \text {. }
$$

From (37) and (42), we have

$$
\begin{gathered}
\mathcal{A}_{2}=\left(\sum _ { u _ { w _ { k } } } \sum _ { \mathbf { c } \in \{ 0 , 1 \} ^ { k } } 2 ^ { k } ( - 1 ) ^ { ( \mathbf { t } _ { 1 } - \mathbf { t } _ { 1 } ^ { \prime } ) \cdot \mathbf { c } } \mathscr { C } \left(\left.f\right|_{\mathbf{u}_{W^{\prime}}=\mathbf{e}, \mathbf{x}_{J_{1}}=\mathbf{c}}+\right.\right. \\
\frac{q}{2} u_{w_{k}} x_{\gamma_{\mathbf{c}}}+\frac{q}{2}\left(\mathbf{t}_{3} \cdot \mathbf{x}_{\mathcal{Z}}\right),\left.f\right|_{\mathbf{u}_{W^{\prime}}=\mathbf{e}, \mathbf{x}_{J_{1}}=\mathbf{c}}+\frac{q}{2} u_{w_{k}} x_{\gamma_{\mathbf{c}}} \\
\left.\left.+\frac{q}{2}\left(\mathbf{t}_{3}^{\prime} \cdot \mathbf{x}_{\mathcal{Z}}\right)\right)(\tau)\right) .
\end{gathered}
$$

Assume, $\left.\mathcal{F}\right|_{\mathbf{x}_{J_{1}}=\mathbf{c}}=\left.f\right|_{\mathbf{u}_{W^{\prime}}=\mathbf{e}, \mathbf{x}_{J_{1}}=\mathbf{c}}$. The following expression present in (44), can be expressed as follows:

$$
\begin{aligned}
& \mathscr{C}\left(\left.f\right|_{\mathbf{u}_{W^{\prime}}=\mathbf{e}, \mathbf{x}_{J_{1}}=\mathbf{c}}+\frac{q}{2} u_{w_{k}} x_{\gamma_{\mathbf{c}}}+\frac{q}{2}\left(\mathbf{t}_{3} \cdot \mathbf{x}_{\mathcal{Z}}\right),\right. \\
& \left.\left.\left.f\right|_{\mathbf{u}_{W^{\prime}}=\mathbf{e}, \mathbf{x}_{J_{1}}=\mathbf{c}}+\frac{q}{2} u_{w_{k}} x_{\gamma_{\mathbf{c}}}+\frac{q}{2}\left(\mathbf{t}_{3}^{\prime} \cdot \mathbf{x}_{\mathcal{Z}}\right)\right)(\tau)\right) \\
& =\sum_{\mathbf{d}^{\prime} \neq \mathbf{d}^{\prime \prime}}(-1)^{\left(\mathbf{t}_{3} \cdot \mathbf{d}^{\prime}-\mathbf{t}_{3}^{\prime} \cdot \mathbf{d}^{\prime \prime}\right)} \mathscr{C}\left(\left.\mathcal{F}\right|_{\mathbf{x}_{J_{1}} \mathbf{x}_{\mathcal{Z}}=\mathbf{c d}^{\prime}}+\frac{q}{2} u_{w_{k}} x_{\gamma_{\mathbf{c}}},\right. \\
& \left.\left.\left.\mathcal{F}\right|_{\mathbf{x}_{J_{1}} \mathbf{x}_{\mathcal{Z}}=\mathbf{c} \mathbf{d}^{\prime \prime}}+\frac{q}{2} u_{w_{k}} x_{\gamma_{\mathbf{c}}}\right)(\tau)\right) \\
& +\sum_{\mathbf{d}^{\prime}=\mathbf{d}^{\prime \prime}}(-1)^{\left(\mathbf{t}_{3} \cdot \mathbf{d}^{\prime}-\mathbf{t}_{3}^{\prime} \cdot \mathbf{d}^{\prime \prime}\right)} \mathscr{C}\left(\left.\mathcal{F}\right|_{\mathbf{x}_{J_{1}} \mathbf{x}_{\mathcal{Z}}=\mathbf{c \mathbf { d } ^ { \prime }}}+\frac{q}{2} u_{w_{k}} x_{\gamma_{\mathfrak{c}}},\right. \\
& \left.\left.\left.\mathcal{F}\right|_{\mathbf{x}_{J_{1}} \mathbf{x}_{\mathcal{Z}}=\mathbf{c} \mathbf{d}^{\prime \prime}}+\frac{q}{2} u_{w_{k}} x_{\gamma_{\mathbf{c}}}\right)(\tau)\right) \\
& =\sum_{\mathbf{d}^{\prime} \neq \mathbf{d}^{\prime \prime}}(-1)^{\left(\mathbf{t}_{3} \cdot \mathbf{d}^{\prime}-\mathbf{t}_{3}^{\prime} \cdot \mathbf{d}^{\prime \prime}\right)} \mathscr{C}\left(\left.\mathcal{F}\right|_{\mathbf{x}_{J_{1}} \mathbf{x}_{\mathcal{Z}}=\mathbf{c d}^{\prime}}+\frac{q}{2} u_{w_{k}} x_{\gamma_{\mathbf{c}}},\right. \\
& \left.\left.\left.\mathcal{F}\right|_{\mathbf{x}_{J_{1}} \mathbf{x}_{\mathcal{Z}}=\mathbf{c} \mathbf{d}^{\prime \prime}}+\frac{q}{2} u_{w_{k}} x_{\gamma_{\mathbf{c}}}\right)(\tau)\right) \\
& +\sum_{\mathbf{d}^{\prime}}(-1)^{\left(\mathbf{t}_{3}-\mathbf{t}_{3}^{\prime}\right) \cdot \mathbf{d}^{\prime}} \mathscr{A}\left(\left.\mathcal{F}\right|_{\mathbf{x}_{J_{1}} \mathbf{x}_{\mathcal{Z}}=\mathbf{c} \mathbf{d}^{\prime}}+\frac{q}{2} u_{w_{k}} x_{\gamma_{\mathbf{c}}}\right)(\tau) \text {. }
\end{aligned}
$$

The only term associated with restricted vertices $x_{j_{0}}, x_{j_{1}}, \ldots, x_{j_{k-1}}$ and isolated vertices $x_{m-p}, x_{m-p+1}, \ldots, x_{m-1}$ can be expressed as follows:

$\mathcal{G}_{\mathbf{x}_{J_{1}} \mathbf{x} z}=\sum_{r=1}^{k} \sum_{\alpha=0}^{p-1} \sum_{0 \leq \alpha_{0}<\alpha_{1}<\ldots<\alpha_{r}<k} \varrho_{j_{0}, p+\alpha, j_{r}}^{m-p+\alpha} x_{j_{\alpha_{0}}} x_{j_{\alpha_{1}} .} x_{j_{\alpha_{r}}} x_{m-p+\alpha}$, where, we have introduced the term $\mathcal{G}_{\mathbf{x}_{J_{1}} \mathbf{x}_{\mathcal{Z}}}$ in (27). By taking sum over $u_{w_{k}}$ and then using Lemma 6 , the following expression of (46) can be expressed as follows:

$$
\begin{aligned}
& \sum_{u_{w_{k}}} \mathscr{C}\left(\left.\mathcal{F}\right|_{\mathbf{x}_{J_{1}} \mathbf{x}_{\mathcal{Z}}=\mathbf{c} \mathbf{d}^{\prime}}+\frac{q}{2} u_{w_{k}} x_{\gamma_{\mathbf{c}}},\right. \\
& \left.\left.\left.\mathcal{F}\right|_{\mathbf{x}_{J_{1}} \mathbf{x}_{\mathcal{Z}}=\mathbf{c d}^{\prime \prime}}+\frac{q}{2} u_{w_{k}} x_{\gamma_{\mathrm{c}}}\right)(\tau)\right)
\end{aligned}
$$

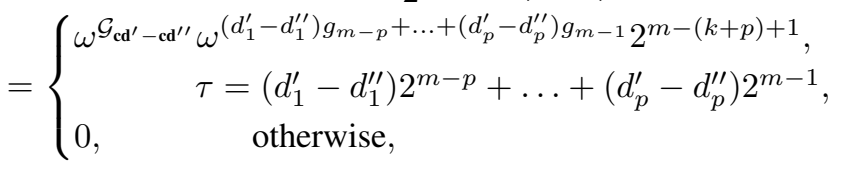

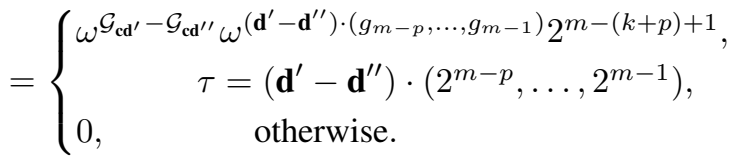

In the above expression $\mathbf{d}^{\prime}$ and $\mathbf{d}^{\prime \prime}$ are two $p$-length binary vectors and $\left(\mathbf{d}^{\prime}-\mathbf{d}^{\prime \prime}\right) \cdot\left(2^{m-p}, \ldots, 2^{m-1}\right)$ takes the value $\tau$. It is possible to get another pair of vectors $\mathbf{d}^{\prime}$ and $\mathbf{d}^{\prime \prime}$ such that $\left(\mathbf{d}^{\prime}-\mathbf{d}^{\prime \prime}\right) \cdot\left(2^{m-p}, \ldots, 2^{m-1}\right)$ also takes the value $\tau$. We assume, for all possible $\mathbf{d}^{\prime}$ and $\mathbf{d}^{\prime \prime}$ in $\{0,1\}^{p},\left(\mathbf{d}^{\prime}-\mathbf{d}^{\prime \prime}\right) \cdot\left(2^{m-p}, \ldots, 2^{m-1}\right)$ takes the integer values $\tau_{1}, \tau_{2}, \ldots, \tau_{\varsigma}$, where $0 \leq \varsigma<3^{p}$. Therefore, we define $\mathcal{K}_{i}=$ $\left\{\left(\mathbf{d}^{\prime}, \mathbf{d}^{\prime \prime}\right): \mathbf{d}^{\prime} \neq \mathbf{d}^{\prime \prime},\left(\mathbf{d}^{\prime}-\mathbf{d}^{\prime \prime}\right) \cdot\left(2^{m-p}, \ldots, 2^{m-1}\right)=\tau_{i}\right\}$, for $i=1,2, \ldots, \varsigma$. From (46) and (47), we have

$$
\begin{aligned}
& \sum_{u_{w_{k}}} \mathscr{C}\left(\left.f\right|_{\mathbf{u}_{W^{\prime}}=\mathbf{e}, \mathbf{x}_{J_{1}}=\mathbf{c}}+\frac{q}{2} u_{w_{k}} x_{\gamma_{\mathbf{c}}}+\frac{q}{2}\left(\mathbf{t}_{3} \cdot \mathbf{x}_{\mathcal{Z}}\right),\right. \\
& \left.\left.\left.f\right|_{\mathbf{u}_{W^{\prime}}=\mathbf{e}, \mathbf{x}_{J_{1}}=\mathbf{c}}+\frac{q}{2} u_{w_{k}} x_{\gamma_{\mathbf{c}}}+\frac{q}{2}\left(\mathbf{t}_{3}^{\prime} \cdot \mathbf{x}_{\mathcal{Z}}\right)\right)(\tau)\right) \\
& =\sum_{\mathbf{d}^{\prime} \neq \mathbf{d}^{\prime \prime}}(-1)^{\left(\mathbf{t}_{3} \cdot \mathbf{d}^{\prime}-\mathbf{t}_{3}^{\prime} \cdot \mathbf{d}^{\prime \prime}\right)} \mathscr{C}\left(\left.\mathcal{F}\right|_{\mathbf{x}_{J_{1}} \mathbf{x}_{\mathcal{Z}}=\mathbf{c \mathbf { d } ^ { \prime }}}+\frac{q}{2} u_{w_{k}} x_{\gamma_{\mathbf{c}}},\right. \\
& \left.\left.\left.\mathcal{F}\right|_{\mathbf{x}_{J_{1}} \mathbf{x}_{\mathcal{Z}}=\mathbf{c d}^{\prime \prime}}+\frac{q}{2} u_{w_{k}} x_{\gamma_{c}}\right)(\tau)\right) \\
& =\sum_{i=1}^{\varsigma} \sum_{\left(\mathbf{d}^{\prime}, \mathbf{d}^{\prime \prime}\right) \in \mathcal{K}_{i}}(-1)^{\left(\mathbf{t}_{3} \cdot \mathbf{d}^{\prime}-\mathbf{t}_{3}^{\prime} \cdot \mathbf{d}^{\prime \prime}\right)} \mathscr{C}\left(\left.\mathcal{F}\right|_{\mathbf{x}_{J_{1}} \mathbf{x}_{\mathcal{Z}}=\mathbf{c d}^{\prime}}+\frac{q}{2} u_{w_{k}} x_{\gamma_{\mathrm{c}}},\right. \\
& \left.\left.\left.\mathcal{F}\right|_{\mathbf{x}_{J_{1}} \mathbf{x}_{\mathcal{Z}}=\mathbf{c} \mathbf{d}^{\prime \prime}}+\frac{q}{2} u_{w_{k}} x_{\gamma_{\mathrm{c}}}\right)(\tau)\right)
\end{aligned}
$$

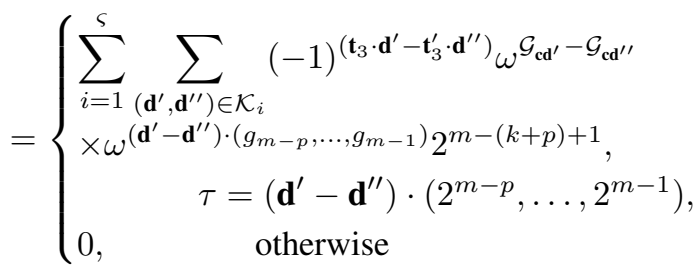

$$
\begin{aligned}
& =\left\{\begin{array}{c}
\sum_{\left(\mathbf{d}^{\prime}, \mathbf{d}^{\prime \prime}\right) \in \mathcal{K}_{i}}(-1)^{\left(\mathbf{t}_{3} \cdot \mathbf{d}^{\prime}-\mathbf{t}_{3}^{\prime} \cdot \mathbf{d}^{\prime \prime}\right)} \omega^{\mathcal{G}_{\mathbf{c d}^{\prime}}-\mathcal{G}_{\mathbf{c d}^{\prime \prime}}} \\
\times \omega^{\left(\mathbf{d}^{\prime}-\mathbf{d}^{\prime \prime}\right) \cdot\left(g_{m-p}, \ldots, g_{m-1}\right)} 2^{m-(k+p)+1}, \\
\tau=\tau_{i}, i=1,2, \ldots, \varsigma \\
0, \quad \text { otherwise. }
\end{array}\right.
\end{aligned}
$$


For each of $\mathbf{c} \in\{0,1\}^{k}, G\left(\left.\mathcal{F}\right|_{\mathbf{x}_{J_{1}} \mathbf{x}_{\mathcal{Z}}=\mathbf{c d}^{\prime \prime}}\right)$ is a path over the vertices specified in $J_{1}^{\prime}$. Therefore,

$\sum_{u_{w_{k}}} \mathscr{A}\left(\left.\mathcal{F}\right|_{\mathbf{x}_{J_{1}} \mathbf{x}_{\mathcal{Z}}=\mathbf{c d}^{\prime}}+\frac{q}{2} u_{w_{k}} x_{\gamma_{\mathrm{c}}}\right)(\tau)=\left\{\begin{array}{l}2^{m-k-p+1}, \tau=0, \\ 0, \quad \text { othewise. }\end{array}\right.$

Therefore, from (45), (46), (48), and (49), we have

$$
\begin{aligned}
& \mathcal{A}_{2}=\left(\sum _ { u _ { w _ { k } } } \sum _ { \mathbf { c } \in \{ 0 , 1 \} ^ { k } } 2 ^ { k } ( - 1 ) ^ { ( \mathbf { t } _ { 1 } - \mathbf { t } _ { 1 } ^ { \prime } ) \cdot \mathbf { c } } \mathscr { C } \left(\left.f\right|_{\mathbf{u}_{W^{\prime}}=\mathbf{e}, \mathbf{x}_{J_{1}}=\mathbf{c}}+\right.\right. \\
& \frac{q}{2} u_{w_{k}} x_{\gamma_{\mathbf{c}}}+\frac{q}{2}\left(\mathbf{t}_{3} \cdot \mathbf{x}_{\mathcal{Z}}\right), \\
& \left.f\right|_{\mathbf{u}_{W^{\prime}}=\mathbf{e}, \mathbf{x}_{J_{1}}=\mathbf{c}}+\frac{q}{2} u_{w_{k}} x_{\gamma_{\mathbf{c}}} \\
& \left.\left.+\frac{q}{2}\left(\mathbf{t}_{3}^{\prime} \cdot \mathbf{x}_{\mathcal{Z}}\right)\right)(\tau)\right)
\end{aligned}
$$

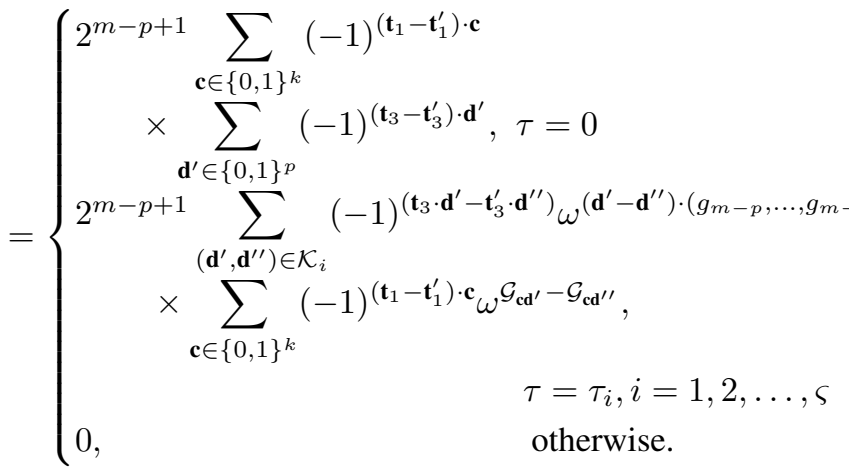

From (38), (40), and (41), we have

$$
\begin{aligned}
& \mathscr{C}\left(\psi\left(S_{t}\right), \psi\left(S_{t^{\prime}}\right)\right)(\tau) \\
& =\sum_{\mathbf{u}_{W^{\prime}}=\mathbf{e}}(-1)^{\left(\mathbf{t}_{2}-\mathbf{t}_{2}^{\prime}\right) \cdot \mathbf{e}} \mathcal{A} \\
& =\left\{\begin{array}{l}
2^{m-p+1} \sum_{\mathbf{u}_{W^{\prime}}=\mathbf{e}}(-1)^{\left(\mathbf{t}_{2}-\mathbf{t}_{2}^{\prime}\right) \cdot \mathbf{e}} \sum_{\mathbf{c} \in\{0,1\}^{k}}(-1)^{\left(\mathbf{t}_{1}-\mathbf{t}_{1}^{\prime}\right) \cdot \mathbf{c}} \\
\times \sum_{\mathbf{d}^{\prime} \in\{0,1\}^{p}}(-1)^{\left(\mathbf{t}_{3}-\mathbf{t}_{3}^{\prime}\right) \cdot \mathbf{d}^{\prime}}, \tau=0 \\
2^{m-p+1}(-1)^{\left(\mathbf{t}_{2}-\mathbf{t}_{2}^{\prime}\right) \cdot \mathbf{e}} \\
\times \sum_{\left(\mathbf{d}^{\prime}, \mathbf{d}^{\prime \prime}\right) \in \mathcal{K}_{i}}^{\mathbf{u}_{W^{\prime}}=\mathbf{e}}(-1)^{\left(\mathbf{t}_{3} \cdot \mathbf{d}^{\prime}-\mathbf{t}_{3}^{\prime} \cdot \mathbf{d}^{\prime \prime}\right)} \omega^{\left(\mathbf{d}^{\prime}-\mathbf{d}^{\prime \prime}\right) \cdot\left(g_{m-p}, \ldots, g_{m-1}\right)} \\
\times \sum_{\mathbf{c} \in\{0,1\}^{k}}^{(-1)^{\left(\mathbf{t}_{1}-\mathbf{t}_{1}^{\prime}\right) \cdot \mathbf{c}} \omega^{\mathcal{G}_{\mathbf{c}^{\prime}}-\mathcal{G}_{\mathbf{c d}} \mathbf{d}^{\prime \prime}},}
\end{array}\right. \\
& \tau=\tau_{i}, i=1,2, \ldots, \varsigma \\
& 0 \text {, } \\
& \text { otherwise. }
\end{aligned}
$$

Similarly, we can show that

$$
\begin{aligned}
& \mathscr{C}\left(\psi^{*}\left(\bar{S}_{t}\right), \psi^{*}\left(\bar{S}_{t^{\prime}}\right)\right)(\tau) \\
& =\sum_{\mathbf{u}_{W^{\prime}}=\mathbf{e}}(-1)^{\left(\mathbf{t}_{2}-\mathbf{t}_{2}^{\prime}\right) \cdot \mathbf{e}} \mathcal{A} \\
& =\left\{\begin{array}{l}
2^{m-p+1} \sum_{\mathbf{u}_{W^{\prime}}=\mathbf{e}}(-1)^{\left(\mathbf{t}_{2}-\mathbf{t}_{2}^{\prime}\right) \cdot \mathbf{e}} \sum_{\mathbf{c} \in\{0,1\}^{k}}(-1)^{\left(\mathbf{t}_{1}-\mathbf{t}_{1}^{\prime}\right) \cdot \mathbf{c}} \\
\times \sum_{\mathbf{d}^{\prime} \in\{0,1\}^{p}}(-1)^{\left(\mathbf{t}_{3}-\mathbf{t}_{3}^{\prime}\right) \cdot \mathbf{d}^{\prime}}, \tau=0 \\
2^{m-p+1}(-1)^{\left(\mathbf{t}_{2}-\mathbf{t}_{2}^{\prime}\right) \cdot \mathbf{e}} \\
\times \sum_{\left(\mathbf{d}^{\prime}, \mathbf{d}^{\prime \prime}\right) \in \mathcal{K}_{i}}^{\mathbf{u}_{W^{\prime}}=\mathbf{e}}(-1)^{\left(\mathbf{t}_{3} \cdot \mathbf{d}^{\prime}-\mathbf{t}_{3}^{\prime} \cdot \mathbf{d}^{\prime \prime}\right)} \omega^{\left(\mathbf{d}^{\prime}-\mathbf{d}^{\prime \prime}\right) \cdot\left(g_{m-p}, \ldots, g_{m-1}\right)} \\
\times \sum_{\mathbf{c} \in\{0,1\}^{k}}^{(-1)^{\left(\mathbf{t}_{1}-\mathbf{t}_{1}^{\prime}\right) \cdot \mathbf{c}} \omega^{\mathcal{G}_{\mathbf{c o d}^{\prime}}-\mathcal{G}_{\mathbf{c d}^{\prime \prime}}},}
\end{array}\right. \\
& \tau=\tau_{i}, i=1,2, \ldots, \varsigma \\
& 0 \text {, }
\end{aligned}
$$

Finally, we need to find out $\mathscr{C}\left(\psi\left(S_{t}\right), \psi^{*}\left(\bar{S}_{t}\right)\right)(\tau)$ for all $\tau$. By using (37), $\mathscr{C}\left(\psi\left(S_{t}\right), \psi^{*}\left(\bar{S}_{t}\right)\right)(\tau)$ can be expressed as

$\mathscr{C}\left(\psi\left(S_{t}\right), \psi^{*}\left(\bar{S}_{t^{\prime}}\right)\right)(\tau)$

$=\sum_{\mathbf{u} \in\{0,1\}^{n}} \mathscr{C}\left(g+\eta_{1}+\frac{q}{2}\left(\mathbf{t}_{1} \cdot \mathbf{x}_{J_{1}}+\mathbf{t}_{2} \cdot \mathbf{u}_{W^{\prime}}+\mathbf{t}_{3} \cdot \mathbf{x}_{\mathcal{Z}}\right)\right.$,

$\left.\tilde{g}+\eta_{2}+\frac{q}{2}\left(\mathbf{t}_{1}^{\prime} \cdot \overline{\mathbf{x}}_{J_{1}}+\mathbf{t}_{2}^{\prime} \cdot \mathbf{u}_{W^{\prime}}+\mathbf{t}_{3}^{\prime} \cdot \overline{\mathbf{x}}_{\mathcal{Z}}\right)\right)(\tau)$

$=\sum_{\mathbf{u}_{W^{\prime}}=\mathbf{e}}(-1)^{\left(\mathbf{t}_{2}+\mathbf{t}_{2}^{\prime}\right) \cdot \mathbf{e}}$

$\times\left(\sum_{\mathbf{u}_{W}, u_{w_{k}}} \mathscr{C}\left(\left.f\right|_{\mathbf{u}_{W^{\prime}}=\mathbf{e}}+\left.h\right|_{\mathbf{u}_{W^{\prime}}=\mathbf{e}}+\eta_{1}+\frac{q}{2}\left(\mathbf{t}_{1} \cdot \mathbf{x}_{J_{1}}+\mathbf{t}_{3} \cdot \mathbf{x}_{\mathcal{Z}}\right)\right.\right.$,

$\left.\left.\left.\tilde{f}^{*}\right|_{\mathbf{u}_{W^{\prime}}=\mathbf{e}}+\eta_{2}+\left.h^{*}\right|_{\mathbf{u}_{W^{\prime}}=\mathbf{e}}+\frac{q}{2}\left(\mathbf{t}_{1}^{\prime} \cdot \overline{\mathbf{x}}_{J_{1}}+\mathbf{t}_{3}^{\prime} \cdot \overline{\mathbf{x}}_{\mathcal{Z}}\right)\right)(\tau)\right)$

$=\sum_{\mathbf{u}_{W^{\prime}}=\mathbf{e}}(-1)^{\left(\mathbf{t}_{2}-\mathbf{t}_{2}^{\prime}\right) \cdot \mathbf{e}} \mathcal{A}_{3}$,

where

$\mathcal{A}_{3}$

$$
\begin{aligned}
& =\sum_{\mathbf{u}_{W}, u_{w_{k}}} \mathscr{C}\left(\left.f\right|_{\mathbf{u}_{W^{\prime}}=\mathbf{e}}+\left.h\right|_{\mathbf{u}_{W^{\prime}}=\mathbf{e}}+\eta_{1}+\frac{q}{2}\left(\mathbf{t}_{1} \cdot \mathbf{x}_{J_{1}}+\mathbf{t}_{3} \cdot \mathbf{x}_{\mathcal{Z}}\right),\right. \\
& \left.\left.\tilde{f}^{*}\right|_{\mathbf{u}_{W^{\prime}}=\mathbf{e}}+\eta_{2}+\left.h^{*}\right|_{\mathbf{u}_{W^{\prime}}=\mathbf{e}}+\frac{q}{2}\left(\mathbf{t}_{1}^{\prime} \cdot \overline{\mathbf{x}}_{J_{1}}+\mathbf{t}_{3}^{\prime} \cdot \overline{\mathbf{x}}_{\mathcal{Z}}\right)\right)(\tau) \\
& =\sum_{\mathbf{u}_{W} u_{w_{k}}=\mathbf{b} b} \mathscr{C}\left(\left.f\right|_{\mathbf{u}_{W^{\prime}}, \mathbf{u}_{W} u_{w_{k}}=\mathbf{e}, \mathbf{b} b}+\left.h\right|_{\mathbf{u}_{W^{\prime}}, \mathbf{u}_{W} u_{w_{k}}=\mathbf{e}, \mathbf{b} b}\right. \\
& \quad+\left.\eta_{1}\right|_{\mathbf{u}_{W} u_{w_{k}}=\mathbf{b} b}+\frac{q}{2}\left(\mathbf{t}_{1} \cdot \mathbf{x}_{J_{1}}+\mathbf{t}_{3} \cdot \mathbf{x}_{\mathcal{Z}}\right), \\
& \left.\tilde{f}^{*}\right|_{\mathbf{u}_{W^{\prime}}, \mathbf{u}_{W} u_{w_{k}}=\mathbf{e}, \mathbf{b} b}+\left.h^{*}\right|_{\mathbf{u}_{W^{\prime}}, \mathbf{u}_{W} u_{w_{k}}=\mathbf{e}, \mathbf{b} b} \\
& \left.+\left.\eta_{2}\right|_{\mathbf{u}_{W} u_{w_{k}}=\mathbf{b} b}+\frac{q}{2}\left(\mathbf{t}_{1}^{\prime} \cdot \overline{\mathbf{x}}_{J_{1}}+\mathbf{t}_{3}^{\prime} \cdot \overline{\mathbf{x}}_{\mathcal{Z}}\right)\right)(\tau)
\end{aligned}
$$




$$
\begin{aligned}
& =\sum_{\mathbf{u}_{W} u_{w_{k}}=\mathbf{b} b} \omega^{\left.2 h\right|_{\mathbf{u}_{W^{\prime}}, \mathbf{u}_{W} u_{w_{k}}=\mathbf{e}, \mathbf{b} b}} \mathscr{C}\left(\left.f\right|_{\mathbf{u}_{W^{\prime}}, \mathbf{u}_{W} u_{w_{k}}=\mathbf{e}, \mathbf{b} b}\right. \\
& +\left.\eta_{1}\right|_{\mathbf{u}_{W} u_{w_{k}}=\mathbf{b} b}+\frac{q}{2}\left(\mathbf{t}_{1} \cdot \mathbf{x}_{J_{1}}+\mathbf{t}_{3} \cdot \mathbf{x}_{\mathcal{Z}}\right) \\
& \left.\tilde{f}^{*}\right|_{\mathbf{u}_{W^{\prime}}, \mathbf{u}_{W} u_{w_{k}}=\mathbf{e}, \mathbf{b} b} \\
& \left.+\left.\eta_{2}\right|_{\mathbf{u}_{W} u_{w_{k}}=\mathbf{b} b}+\frac{q}{2}\left(\mathbf{t}_{1}^{\prime} \cdot \overline{\mathbf{x}}_{J_{1}}+\mathbf{t}_{3}^{\prime} \cdot \overline{\mathbf{x}}_{\mathcal{Z}}\right)\right)(\tau) .
\end{aligned}
$$

From (25) and (53), we have

$$
\begin{aligned}
\mathcal{A}_{3}= & \omega^{2 \delta} \sum_{\mathbf{u}_{W} u_{w_{k}}=\mathbf{b} b} \mathscr{C}\left(\left.f\right|_{\mathbf{u}_{W^{\prime}}, \mathbf{u}_{W} u_{w_{k}}=\mathbf{e}, \mathbf{b} b}\right. \\
& +\left.\eta_{1}\right|_{\mathbf{u}_{W} u_{w_{k}}=\mathbf{b} b}+\frac{q}{2}\left(\mathbf{t}_{1} \cdot \mathbf{x}_{J_{1}}+\mathbf{t}_{3} \cdot \mathbf{x}_{\mathcal{Z}}\right), \\
& \left.\tilde{f}^{*}\right|_{\mathbf{u}_{W^{\prime}}, \mathbf{u}_{W} u_{w_{k}}=\mathbf{e}, \mathbf{b} b} \\
& \left.+\left.\eta_{2}\right|_{\mathbf{u}_{W} u_{w_{k}}=\mathbf{b} b}+\frac{q}{2}\left(\mathbf{t}_{1}^{\prime} \cdot \overline{\mathbf{x}}_{J_{1}}+\mathbf{t}_{3}^{\prime} \cdot \overline{\mathbf{x}}_{\mathcal{Z}}\right)\right)(\tau) \\
& =\omega^{2 \delta} \mathcal{A}_{4},
\end{aligned}
$$

where,

$$
\begin{array}{rl}
\mathcal{A}_{4}=\sum_{\mathbf{u}_{W} u_{w_{k}}=\mathbf{b} b} & \mathscr{C}\left(\left.f\right|_{\mathbf{u}_{W^{\prime}}, \mathbf{u}_{W} u_{w_{k}}=\mathbf{e}, \mathbf{b} b}\right. \\
& +\left.\eta_{1}\right|_{\mathbf{u}_{W} u_{w_{k}}=\mathbf{b} b}+\frac{q}{2}\left(\mathbf{t}_{1} \cdot \mathbf{x}_{J_{1}}+\mathbf{t}_{3} \cdot \mathbf{x}_{\mathcal{Z}}\right), \\
\left.\tilde{f}^{*}\right|_{\mathbf{u}_{W^{\prime}}, \mathbf{u}_{W} u_{w_{k}}=\mathbf{e}, \mathbf{b} b} & \\
& \left.+\left.\eta_{2}\right|_{\mathbf{u}_{W} u_{w_{k}}=\mathbf{b} b}+\frac{q}{2}\left(\mathbf{t}_{1}^{\prime} \cdot \overline{\mathbf{x}}_{J_{1}}+\mathbf{t}_{3}^{\prime} \cdot \overline{\mathbf{x}}_{\mathcal{Z}}\right)\right)(\tau) .
\end{array}
$$

Assume, $\mathcal{F}_{1}=\left.f\right|_{\mathbf{u}_{W^{\prime}}, \mathbf{u}_{W} u_{w_{k}}=\mathbf{e}, \mathbf{b} b}$. From, (37), we have

$$
\begin{aligned}
&\left.\eta_{1}\right|_{\mathbf{u}_{W} u_{w_{k}}=\mathbf{b} b}=\frac{q}{2}\left(\mathbf{b} \cdot \mathbf{x}_{J_{1}}+b x_{\gamma_{\mathbf{c}}}\right), \\
&\left.\eta_{2}\right|_{\mathbf{u}_{W} u_{w_{k}}=\mathbf{b} b}=\frac{q}{2}\left(\mathbf{b} \cdot \overline{\mathbf{x}}_{J_{1}}+\bar{b} x_{\gamma_{\mathbf{c}}}\right) .
\end{aligned}
$$

From (57), (56), and substituting $\mathcal{F}_{1}=\left.f\right|_{\mathbf{u}_{W^{\prime}}, \mathbf{u}_{W} u_{w_{k}}=\mathbf{e}, \mathbf{b} b}$ in (56), we have

$$
\begin{aligned}
\mathcal{A}_{4}=\sum_{\mathbf{u}_{W} u_{w_{k}}=\mathbf{b} b} \mathscr{C}\left(\mathcal{F}_{1}+\frac{q}{2}\left(\mathbf{b} \cdot \mathbf{x}_{J_{1}}+b x_{\gamma_{\mathbf{c}}}\right)\right. \\
+\frac{q}{2}\left(\mathbf{t}_{1} \cdot \mathbf{x}_{J_{1}}+\mathbf{t}_{3} \cdot \mathbf{x}_{\mathcal{Z}}\right), \\
\tilde{\mathcal{F}}_{1}^{*}+\frac{q}{2}\left(\mathbf{b} \cdot \overline{\mathbf{x}}_{J_{1}}+\bar{b} x_{\gamma_{\mathbf{c}}}\right) \\
\left.+\frac{q}{2}\left(\mathbf{t}_{1}^{\prime} \cdot \overline{\mathbf{x}}_{J_{1}}+\mathbf{t}_{3}^{\prime} \cdot \overline{\mathbf{x}}_{\mathcal{Z}}\right)\right)(\tau) .
\end{aligned}
$$

For each $\mathbf{c} \in\{0,1\}^{k}, G\left(\left.\mathcal{F}_{1}\right|_{\mathbf{x}_{J_{1}}=\mathbf{c}}\right)$ contains a path over the vertices specified in $J_{1}^{\prime}$ and $p$ isolated vertices labeled $m-$ $p, m-p+1, \ldots, m-1$. Therefore, by employing Lemma 4 in (58), we have

$$
\begin{aligned}
& \mathcal{A}_{4}= \sum_{\mathbf{u}_{W} u_{w_{k}}=\mathbf{b} b} \mathscr{C}\left(\mathcal{F}_{1}+\frac{q}{2}\left(\mathbf{b} \cdot \mathbf{x}_{J_{1}}+b x_{\gamma_{\mathbf{c}}}\right)\right. \\
&+\frac{q}{2}\left(\mathbf{t}_{1} \cdot \mathbf{x}_{J_{1}}+\mathbf{t}_{3} \cdot \mathbf{x}_{\mathcal{Z}}\right), \\
& \tilde{\mathcal{F}}_{1}^{*}+\frac{q}{2}\left(\mathbf{b} \cdot \overline{\mathbf{x}}_{J_{1}}+\bar{b} x_{\gamma_{\mathbf{c}}}\right) \\
&\left.\quad+\frac{q}{2}\left(\mathbf{t}_{1}^{\prime} \cdot \overline{\mathbf{x}}_{J_{1}}+\mathbf{t}_{3}^{\prime} \cdot \overline{\mathbf{x}}_{\mathcal{Z}}\right)\right)(\tau) \\
&=0 \forall \tau .
\end{aligned}
$$

From, (53), (55), and (59), we have

$$
\mathscr{C}\left(\psi\left(S_{t}\right), \psi^{*}\left(\bar{S}_{t^{\prime}}\right)\right)(\tau)=0 \forall \tau
$$

We have defined before

$$
\mathcal{K}_{i}=\left\{\left(\mathbf{d}^{\prime}, \mathbf{d}^{\prime \prime}\right): \mathbf{d}^{\prime} \neq \mathbf{d}^{\prime \prime},\left(\mathbf{d}^{\prime}-\mathbf{d}^{\prime \prime}\right) \cdot\left(2^{m-p}, \ldots, 2^{m-1}\right)=\tau_{i}\right\}
$$

for $i=1,2, \ldots, \varsigma$. Now, we shall find out $\min _{i \in\{1,2, \ldots, \varsigma\}}\left|\tau_{i}\right|$.

$$
\begin{aligned}
\left|\tau_{i}\right| & =\left|\left(\mathbf{d}^{\prime}-\mathbf{d}^{\prime \prime}\right) \cdot\left(2^{m-p}, \ldots, 2^{m-1}\right)\right| \\
& =\left|\left(d_{1}^{\prime}-d_{1}^{\prime \prime}\right) 2^{m-p}+\ldots+\left(d_{p}^{\prime}-d_{p}^{\prime \prime}\right) 2^{m-1}\right| \\
& =2^{m-p}\left|\left(d_{1}^{\prime}-d_{1}^{\prime \prime}\right)+\ldots+\left(d_{p}^{\prime}-d_{p}^{\prime \prime}\right) 2^{p-1}\right| \\
& \geq 2^{m-p} .
\end{aligned}
$$

In (61), the equality occurs if we take $\mathbf{d}^{\prime}=(1,0, \ldots, 0)$ and $\mathbf{d}^{\prime \prime}=(0,0, \ldots, 0)$. There can exist another $\mathbf{d}^{\prime}$ and $\mathbf{d}^{\prime \prime}$ for which the equality can also occur. Therefore,

$$
\min _{i \in\{1,2, \ldots, \varsigma\}}\left|\tau_{i}\right|=2^{m-p}
$$

From (51), we have

$$
\mathscr{C}\left(\psi\left(S_{t}\right), \psi\left(S_{t^{\prime}}\right)\right)(\tau)= \begin{cases}2^{m+n}, & \tau=0, t=t^{\prime} \\ 0, & 0<|\tau|<2^{m-p}, t=t^{\prime} \\ 0, & |\tau|<2^{m-p}, t \neq t^{\prime}\end{cases}
$$

From (52), we have

$$
\mathscr{C}\left(\psi\left(\bar{S}_{t}\right), \psi\left(\bar{S}_{t^{\prime}}\right)\right)(\tau)= \begin{cases}2^{m+n}, & \tau=0, t=t^{\prime} \\ 0, & 0<|\tau|<2^{m-p}, t=t^{\prime} \\ 0, & |\tau|<2^{m-p}, t \neq t^{\prime}\end{cases}
$$

Finally, from (60), (63), and (64), we have

$\left\{\psi\left(S_{t}\right): 0 \leq t \leq 2^{n+p-1}-1\right\} \cup\left\{\psi^{*}\left(\bar{S}_{t}\right): 0 \leq t \leq 2^{n+p-1}-1\right\}$,

is a $\left(2^{n+p}, 2^{m-p}\right)-\mathrm{ZCCS}_{2^{n}}^{2^{m}}$.

\section{APPENDIX B \\ PROOF OF Theorem 2}

The $\psi\left(S_{t}\right)$ and $\psi\left(S_{t^{\prime}}\right)$ will be in a same code group if $\mathbf{t}_{1}=$ $\mathbf{t}_{1}^{\prime}, \mathbf{t}_{2}=\mathbf{t}_{2}^{\prime}$, otherwise the codes will be in two different code groups. The term $\mathcal{G}_{\mathbf{x}_{J_{1}} \mathbf{x}_{\mathcal{Z}}}$ is assumed to be zero in Theorem 
2. Therefore, by replacing $\mathcal{G}_{\mathbf{c d}^{\prime}}=\mathcal{G}_{\mathbf{c d}^{\prime \prime}}=0$ in (51) and from (62), we have

$$
\begin{aligned}
& \mathscr{C}\left(\psi\left(S_{t}\right), \psi\left(S_{t^{\prime}}\right)\right)(\tau) \\
& \left\{\begin{array}{l}
2^{m-p+1} \sum_{\mathbf{u}_{W^{\prime}}=\mathbf{e}}(-1)^{\left(\mathbf{t}_{2}-\mathbf{t}_{2}^{\prime}\right) \cdot \mathbf{e}} \sum_{\mathbf{c} \in\{0,1\}^{k}}(-1)^{\left(\mathbf{t}_{1}-\mathbf{t}_{1}^{\prime}\right) \cdot \mathbf{c}} \\
\times \sum_{\mathbf{d}^{\prime} \in\{0,1\}^{p}}(-1)^{\left(\mathbf{t}_{3}-\mathbf{t}_{3}^{\prime}\right) \cdot \mathbf{d}^{\prime}}, \tau=0 \\
\times \sum_{\left(\mathbf{d}^{\prime}, \mathbf{d}^{\prime \prime}\right) \in \mathcal{K}_{i}}(-1)^{\left(\mathbf{t}_{3} \cdot \mathbf{d}^{\prime}-\mathbf{t}_{3}^{\prime} \cdot \mathbf{d}^{\prime \prime}\right)} \omega^{\left(\mathbf{d}^{\prime}-\mathbf{d}^{\prime \prime}\right) \cdot\left(g_{m-p}, \ldots, g_{m-1}\right)} \\
\times \sum_{\mathbf{c} \in\{0,1\}^{k}}(-1)^{\left(\mathbf{t}_{1}-\mathbf{t}_{1}^{\prime}\right) \cdot \mathbf{c}}, \quad \text { otherwise. } \\
0, \quad \text { ot } \tau_{i}^{\prime}, i=1,2, \ldots, \varsigma
\end{array}\right. \\
& =\left\{\begin{array}{l}
2^{m+n}, \tau=0, \mathbf{t}_{1}=\mathbf{t}_{1}^{\prime}, \mathbf{t}_{2}=\mathbf{t}_{2}^{\prime}, \mathbf{t}_{3}=\mathbf{t}_{3}^{\prime}, \\
0, \quad 0<|\tau|<2^{m-p}, \mathbf{t}_{1}=\mathbf{t}_{1}^{\prime}, \mathbf{t}_{2}=\mathbf{t}_{2}^{\prime}, \mathbf{t}_{3}=\mathbf{t}_{3}^{\prime}, \\
0,|\tau|<2^{m-p}, \mathbf{t}_{1}=\mathbf{t}_{1}^{\prime}, \mathbf{t}_{2}=\mathbf{t}_{2}^{\prime}, \mathbf{t}_{3} \neq \mathbf{t}_{3}^{\prime}, \\
0,|\tau|<2^{m}, \mathbf{t}_{1}=\mathbf{t}_{1}^{\prime}, \mathbf{t}_{2} \neq \mathbf{t}_{2}^{\prime}, \mathbf{t}_{3}=\mathbf{t}_{3}^{\prime}, \\
0,|\tau|<2^{m}, \mathbf{t}_{1}=\mathbf{t}_{1}^{\prime}, \mathbf{t}_{2} \neq \mathbf{t}_{2}^{\prime}, \mathbf{t}_{3} \neq \mathbf{t}_{3}^{\prime}, \\
0,|\tau|<2^{m}, \mathbf{t}_{1} \neq \mathbf{t}_{1}^{\prime}, \mathbf{t}_{2}=\mathbf{t}_{2}^{\prime}, \mathbf{t}_{3}=\mathbf{t}_{3}^{\prime}, \\
0,|\tau|<2^{m}, \mathbf{t}_{1} \neq \mathbf{t}_{1}^{\prime}, \mathbf{t}_{2}=\mathbf{t}_{2}^{\prime}, \mathbf{t}_{3} \neq \mathbf{t}_{3}^{\prime}, \\
0,|\tau|<2^{m}, \mathbf{t}_{1} \neq \mathbf{t}_{1}^{\prime}, \mathbf{t}_{2} \neq \mathbf{t}_{2}^{\prime}, \mathbf{t}_{3}=\mathbf{t}_{3}^{\prime}, \\
0,|\tau|<2^{m}, \mathbf{t}_{1} \neq \mathbf{t}_{1}^{\prime}, \mathbf{t}_{2} \neq \mathbf{t}_{2}^{\prime}, \mathbf{t}_{3} \neq \mathbf{t}_{3}^{\prime} .
\end{array}\right.
\end{aligned}
$$

Similarly, from (52), we can show that

$$
\begin{aligned}
& \mathscr{C}\left(\psi^{*}\left(\bar{S}_{t}\right), \psi^{*}\left(\bar{S}_{t^{\prime}}\right)\right)(\tau) \\
& =\left\{\begin{array}{l}
2^{m+n}, \tau=0, \mathbf{t}_{1}=\mathbf{t}_{1}^{\prime}, \mathbf{t}_{2}=\mathbf{t}_{2}^{\prime}, \mathbf{t}_{3}=\mathbf{t}_{3}^{\prime}, \\
0,0<|\tau|<2^{m-p}, \mathbf{t}_{1}=\mathbf{t}_{1}^{\prime}, \mathbf{t}_{2}=\mathbf{t}_{2}^{\prime}, \mathbf{t}_{3}=\mathbf{t}_{3}^{\prime}, \\
0,|\tau|<2^{m-p}, \mathbf{t}_{1}=\mathbf{t}_{1}^{\prime}, \mathbf{t}_{2}=\mathbf{t}_{2}^{\prime}, \mathbf{t}_{3} \neq \mathbf{t}_{3}^{\prime}, \\
0,|\tau|<2^{m}, \mathbf{t}_{1}=\mathbf{t}_{1}^{\prime}, \mathbf{t}_{2} \neq \mathbf{t}_{2}^{\prime}, \mathbf{t}_{3}=\mathbf{t}_{3}^{\prime}, \\
0,|\tau|<2^{m}, \mathbf{t}_{1}=\mathbf{t}_{1}^{\prime}, \mathbf{t}_{2} \neq \mathbf{t}_{2}^{\prime}, \mathbf{t}_{3} \neq \mathbf{t}_{3}^{\prime}, \\
0,|\tau|<2^{m}, \mathbf{t}_{1} \neq \mathbf{t}_{1}^{\prime}, \mathbf{t}_{2}=\mathbf{t}_{2}^{\prime}, \mathbf{t}_{3}=\mathbf{t}_{3}^{\prime}, \\
0,|\tau|<2^{m}, \mathbf{t}_{1} \neq \mathbf{t}_{1}^{\prime}, \mathbf{t}_{2}=\mathbf{t}_{2}^{\prime}, \mathbf{t}_{3} \neq \mathbf{t}_{3}^{\prime}, \\
0,|\tau|<2^{m}, \mathbf{t}_{1} \neq \mathbf{t}_{1}^{\prime}, \mathbf{t}_{2} \neq \mathbf{t}_{2}^{\prime}, \mathbf{t}_{3}=\mathbf{t}_{3}^{\prime}, \\
0,|\tau|<2^{m}, \mathbf{t}_{1} \neq \mathbf{t}_{1}^{\prime}, \mathbf{t}_{2} \neq \mathbf{t}_{2}^{\prime}, \mathbf{t}_{3} \neq \mathbf{t}_{3}^{\prime} .
\end{array}\right.
\end{aligned}
$$

Also, from (60), we have

$$
\mathscr{C}\left(\psi\left(S_{t}\right), \psi^{*}\left(\bar{S}_{t^{\prime}}\right)\right)(\tau)=0 \forall \tau .
$$

By using the results of (66), (67), and (60), the code groups $\mathcal{I}_{0}, \mathcal{I}_{1}, \ldots, \mathcal{I}_{2^{n-1}-1}, \overline{\mathcal{I}}_{0}^{*}, \overline{\mathcal{I}}_{1}^{*}, \ldots, \overline{\mathcal{I}}_{2^{n-1}-1}^{*}$ forms an IGC code set $\mathcal{I}\left(2^{n+p}, 2^{n}, 2^{m}, 2^{m-p}\right)$.

\section{REFERENCES}

[1] A. Rathinakumar and A. K. Chaturvedi, "Complete mutually orthogonal Golay complementary sets from Reed-Muller codes," IEEE Trans. Inf. Theory, vol. 54, no. 3, pp. 1339-1346, Mar. 2008

[2] S. Das, S. Budišin, S. Majhi, Z. Liu, and Y. L. Guan, "A multiplier-free generator for polyphase complete complementary codes," IEEE Trans. Signal Process., vol. 66, no. 5, pp. 1184-1196, Mar. 2018.
[3] P. Sarkar, S. Majhi, and Z. Liu, "Optimal $Z$-complementary code set from generalized Reed-Muller codes," IEEE Trans. Commun., vol. 67, no. 3, pp. 1783-1796, Mar. 2019.

[4] S. Wu and C. Chen, "Optimal Z-complementary sequence sets with good peak-to-average power-ratio property," IEEE Signal Process. Lett., vol. 25, no. 10, pp. 1500-1504, Oct. 2018.

[5] Y. $\mathrm{Li}$ and $\mathrm{C} . \mathrm{Xu}$, "ZCZ aperiodic complementary sequence sets with low column sequence PMEPR," IEEE Commun. Lett., vol. 19, no. 8, pp. 1303-1306, Aug. 2015

[6] S. Das, S. Budiin, S. Majhi, Z. Liu, and Y. L. Guan, "A multiplier-free generator for polyphase complete complementary codes," IEEE Trans. Signal Process., vol. 66, no. 5, pp. 1184-1196, Mar. 2018.

[7] A. R. Adhikary and S. Majhi, "New construction of optimal aperiodic z-complementary sequence sets of odd-lengths," Electron. Lett., July 2019. [Online]. Available: https://digitallibrary.theiet.org/content/journals/10.1049/el.2019.1828

[8] Y. Li, L. Tian, and C. Xu, "Constructions of asymptotically optimal aperiodic quasi-complementary sequence sets," IEEE Trans. Commun., pp. $1-1,2019$

[9] M. Golay, "Complementary series," IRE Trans. Inf. Theory, vol. 7, no. 2, pp. 82-87, Apr. 1961.

[10] C.-C. Tseng and C. Liu, "Complementary sets of sequences," IEEE Trans. Inf. Theory, vol. 18, no. 5, pp. 644-652, Sep. 1972.

[11] J. A. Davis and J. Jedwab, "Peak-to-mean power control in OFDM, Golay complementary sequences, and Reed-Muller codes," IEEE Trans. Inf. Theory, vol. 45, no. 7, pp. 2397-2417, Nov. 1999.

[12] K. G. Paterson, "Generalized Reed-Muller codes and power control in OFDM modulation," IEEE Trans. Inf. Theory, vol. 46, no. 1, pp. 104 120, Jan. 2000.

[13] K. U. Schmidt, "Complementary sets, generalized Reed-Muller codes, and power control for OFDM," IEEE Trans. Inf. Theory, vol. 53, no. 2, pp. 808-814, Feb. 2007.

[14] Z. Liu, Y. L. Guan, and U. Parampalli, "New complete complementary codes for peak-to-mean power control in multi-carrier CDMA," IEEE Trans. Commun., vol. 62, no. 3, pp. 1105-1113, Mar. 2014

[15] Z. Liu, Y. L. Guan, B. C. Ng, and H.-H. Chen, "Correlation and set size bounds of complementary sequences with low correlation zone," IEEE Trans. Commun., vol. 59, no. 12, pp. 3285-3289, Dec. 2011.

[16] Z. Liu, Y. L. Guan, and U. Parampalli, "A new construction of zero correlation zone sequences from generalized Reed-Muller codes," in 2014 IEEE Information Theory Workshop, Nov. 2014, pp. 591-595.

[17] P. Fan, W. Yuan, and Y. Tu, "Z-complementary binary sequences," IEEE Signal Process. Lett., vol. 14, no. 8, pp. 509-512, Aug. 2007.

[18] L. Feng, P. Fan, X. Tang, and K. K. Loo, "Generalized pairwise Zcomplementary codes," IEEE Signal Process. Lett., vol. 15, pp. 377-380, 2008.

[19] J. Li, A. Huang, M. Guizani, and H.-H. Chen, "Inter group complementary codes for interference resistant CDMA wireless communications," IEEE Trans. Wireless Commun., vol. 7, no. 1, pp. 166-174, Jan. 2008.

[20] P. Sarkar, S. Majhi, H. Vettikalladi, and A. S. Mahajumi, "A direct construction of inter-group complementary code set," IEEE Access, pp. $1-1,2018$.

[21] A. Samad, A. R. Adhikary, and S. Majhi, "Receiver design for quasiasynchronous MC-CDMA by using QCSS code," in 2016 International Conference on Communication and Signal Processing (ICCSP), Apr. 2016, pp. 1159-1163. 